TRANSACTIONS OF THE

AMERICAN MATHEMATICAL SOCIETY

Volume 362, Number 12, December 2010, Pages 6293-6324

S 0002-9947(2010)05031-7

Article electronically published on July 13, 2010

\title{
TOTAL CURVATURES OF MODEL SURFACES CONTROL TOPOLOGY OF COMPLETE OPEN MANIFOLDS WITH RADIAL CURVATURE BOUNDED BELOW. II
}

\author{
KEI KONDO AND MINORU TANAKA
}

\begin{abstract}
We prove, as our main theorem, the finiteness of topological type of a complete open Riemannian manifold $M$ with a base point $p \in M$ whose radial curvature at $p$ is bounded from below by that of a non-compact model surface of revolution $\widetilde{M}$ which admits a finite total curvature and has no pair of cut points in a sector. Here a sector is, by definition, a domain cut off by two meridians emanating from the base point $\tilde{p} \in \widetilde{M}$. Notice that our model $\widetilde{M}$ does not always satisfy the diameter growth condition introduced by Abresch and Gromoll. In order to prove the main theorem, we need a new type of the Toponogov comparison theorem. As an application of the main theorem, we present a partial answer to Milnor's open conjecture on the fundamental group of complete open manifolds.
\end{abstract}

\section{INTRODUCTION}

Most comparison theorems which appear in differential geometry originated from the Sturm comparison theorem (see $[\mathrm{St}]$ or $[\mathrm{Hr}]$ ). Bonnet $[\mathrm{Bo}$. would be the first researcher who applied the Sturm comparison theorem to differential geometry. In 1855, he proved that the diameter of a compact surface does not exceed $\pi / \sqrt{\Lambda}$ if the Gaussian curvature of the surface is greater than a positive constant $\Lambda$.

In 1932, Schoenberg [Sc formulated the Sturm comparison theorem by using the sectional curvatures of Riemannian manifolds and generalized Bonnet's theorem to any Riemannian manifold. Therefore, it took more than 70 years to generalize Bonnet's theorem above, though von Mangoldt [Ma also applied the Sturm comparison theorem to differential geometry in 1881 .

In 1951, Rauch succeeded in comparing the lengths of Jacobi fields along geodesics in differential Riemannian manifolds and proved a well-known theorem, which is called the Rauch comparison theorem. Thus its special case is Schoenberg's theorem above. As an application of the Rauch comparison theorem, he proved a (topological) sphere theorem in $[\underline{R}$. After this work, various kinds of sphere theorems have been proved (cf. [K1], [K2, [B], GS], Sh2], and so on).

In 1959, Toponogov To1], [To2] generalized the Rauch comparison theorem as a global version of the Rauch comparison theorem. He compared the angles of geodesic triangles in a Riemannian manifold and those in a complete 2-dimensional

Received by the editors December 17, 2007 and, in revised form, June 30, 2008.

2010 Mathematics Subject Classification. Primary 53C21; Secondary 53C22.

Key words and phrases. Toponogov's comparison theorem, cut locus, geodesic, radial curvature, total curvature.

(C)2010 American Mathematical Society Reverts to public domain 28 years from publication 6293 
Riemannian manifold of constant Gaussian curvature, which is well known as the Alexandrov-Toponogov comparison theorem (abbreviated to just the "Toponogov" comparison theorem). The Toponogov comparison theorem is now a very powerful tool for investigating global structures of Riemannian manifolds (cf. [CG], GS], G1, and so on).

Some researchers tried to generalize the Toponogov comparison theorem. In 1985, Abresch A generalized the Toponogov comparison theorem by a non-compact model surface of revolution with non-positive Gaussian curvature. He would be the first researcher who succeeded in generalizing it in a rather satisfactory form, that is, in the radial curvature geometry.

We will now introduce the radial curvature geometry for pointed complete open Riemannian manifolds. We call a complete open 2-dimensional Riemannian manifold $(\widetilde{M}, \tilde{p})$ with a base point $\tilde{p} \in \widetilde{M}$ a non-compact model surface of revolution if its Riemannian metric $d \tilde{s}^{2}$ is expressed in terms of geodesic polar coordinates around $\tilde{p}$ as

$$
d \tilde{s}^{2}=d t^{2}+f(t)^{2} d \theta^{2}, \quad(t, \theta) \in(0, \infty) \times \mathbb{S}_{\tilde{p}}^{1}
$$

Here, $f:(0, \infty) \longrightarrow \mathbb{R}$ is a positive smooth function which is extensible to a smooth odd function around 0 , and $\mathbb{S}_{\tilde{p}}^{1}:=\left\{v \in T_{\tilde{p}} \widetilde{M} \mid\|v\|=1\right\}$. The function $G \circ \tilde{\gamma}:[0, \infty) \longrightarrow \mathbb{R}$ is called the radial curvature function of $(\widetilde{M}, \tilde{p})$, where we denote by $G$ the Gaussian curvature of $\widetilde{M}$, and by $\tilde{\gamma}$ any meridian emanating from $\tilde{p}=\tilde{\gamma}(0)$. Observe that $f$ satisfies the differential equation

$$
f^{\prime \prime}(t)+G(\tilde{\gamma}(t)) f(t)=0
$$

with initial conditions $f(0)=0$ and $f^{\prime}(0)=1$. The $n$-dimensional model surfaces of revolution are defined similarly, and they are completely classified in [KK].

Let $(M, p)$ be a complete open $n$-dimensional Riemannian manifold with a base point $p \in M$. We say that $(M, p)$ has radial curvature at the base point $p$ bounded from below by that of a non-compact model surface of revolution $(\widetilde{M}, \tilde{p})$ if, along every unit speed minimal geodesic $\gamma:[0, a) \longrightarrow M$ emanating from $p=\gamma(0)$, its sectional curvature $K_{M}$ satisfies

$$
K_{M}\left(\sigma_{t}\right) \geq G(\tilde{\gamma}(t))
$$

for all $t \in[0, a)$ and all 2-dimensional linear spaces $\sigma_{t}$ spanned by $\gamma^{\prime}(t)$ and a tangent vector to $M$ at $\gamma(t)$.

For example, if the Riemannian metric of $\widetilde{M}$ is $d t^{2}+t^{2} d \theta^{2}$, or $d t^{2}+\sinh ^{2} t d \theta^{2}$, then $G(\tilde{\gamma}(t))=0$, or $G(\tilde{\gamma}(t))=-1$, respectively. Furthermore, the radial curvature may change signs. Moreover, we can employ a model surface of revolution, as a comparison surface, satisfying $\lim _{t \rightarrow \infty} G(\tilde{\gamma}(t))=-\infty$. Thus, it is very natural as a generalization of conventional comparison geometry to make use of a model surface of revolution instead of a complete simply connected surface of constant Gaussian curvature.

In 2003, the Toponogov comparison theorem was generalized by Itokawa, Machigashira, and Shiohama, by using a von Mangoldt surface of revolution as an $(\widetilde{M}, \tilde{p})$, in a very satisfactory form; i.e., their theorem contains the original Toponogov comparison theorem as a corollary (see IMS, Theorem 1.3]). Here, a von Mangoldt surface of revolution is, by definition, a model surface of revolution whose radial curvature function is non-increasing on $[0, \infty)$. Paraboloids and 2 -sheeted 
hyperboloids are typical examples of a von Mangoldt surface of revolution. An untypical example of a von Mangoldt surface of revolution is found in [KT1, Example $1.2]$, where its radial curvature function $G(\tilde{\gamma}(t))$ changes signs on $[0, \infty)$. We refer to T1 for other examples of a von Mangoldt surface of revolution. Thus, a von Mangoldt surface of revolution is a very common model as a reference space. We refer to [KO] and [KT1] for applications of the Itokawa-Machigashira-Shiohama comparison theorem.

Poincaré $\underline{\mathrm{PO}}$ first introduced the notion of the cut locus for surfaces in 1905 . He claimed that the endpoints of the cut locus are cusps of the conjugate locus turned to the starting point. We have explicitly determined such structures of model surfaces of revolution whose Gaussian curvature is monotonic along a subarc of a meridian (see [T1, GMST], SiT1, and [SiT2]). For example, the cut locus Cut $(\tilde{z})$ to each point $\tilde{z} \in \widetilde{M} \backslash\{\tilde{p}\}$ of a non-compact von Mangoldt surface of revolution $(\widetilde{M}, \tilde{p})$ is either an empty set, or a ray properly contained in the meridian $\theta^{-1}(\theta(\tilde{z})+\pi)$ laying opposite to $\tilde{z}$, and that the endpoint of $\operatorname{Cut}(\tilde{z})$ is the first conjugate point to $\tilde{z}$ along the minimal geodesic from $\tilde{z}$ sitting in $\theta^{-1}(\theta(\tilde{z})) \cup \theta^{-1}(\theta(\tilde{z})+\pi)$ (see T1, Main Theorem]). In particular, any non-compact von Mangoldt surface of revolution has no pair of cut points in the sector $\widetilde{V}(\pi)$, where we define a sector

$$
\widetilde{V}(\delta):=\{\tilde{x} \in \widetilde{M} \mid 0<\theta(\tilde{x})<\delta\}
$$

for each constant number $\delta>0$.

However, we emphasize that the cut locus on a model surface of revolution need not be connected. A model surface of revolution with a disconnected cut locus has been constructed in [T2, Section 2]. We also note that Gluck and Singer GlSi] constructed a smooth, but non-analytic surface of revolution embedded in $\mathbb{R}^{3}$ such that the cut locus of a point on the surface admits a branch point with infinite order, even one of strictly positive Gaussian curvature, so that its cut locus has also infinitely many endpoints.

Thus, it is not difficult to establish the Toponogov comparison theorem for a model surface of revolution admitting a very simple structure of the cut locus at each point as seen in IMS, Theorem 1.3]. However, it is very difficult to establish the Toponogov comparison theorem for an arbitrary model surface of revolution. The cause of the difficulty lies in the complexity of cut loci of model surfaces of revolution as stated above. Therefore, the structure of cut loci of model surfaces of revolution allows a generalization of the Toponogov comparison theorem in radial curvature geometry.

In this article, we are concerned with a generalization of the Toponogov comparison theorem to the radial curvature geometry. That is, we need a new type of the Toponogov comparison theorem (Theorem 4.12 in Section 44) in order to prove our main theorem:

A new type of Toponogov comparison theorem. Let $(M, p)$ be a complete open Riemannian n-manifold $M$ whose radial curvature at the base point $p$ is bounded from below by that of a non-compact model surface of revolution $(\widetilde{M}, \tilde{p})$. Assume that $\widetilde{M}$ admits a sector $\widetilde{V}\left(\delta_{0}\right)$ for some $\delta_{0} \in(0, \pi)$ which has no pair of cut points. Then, for every geodesic triangle $\triangle(p x y)$ in $M$ with $\angle(x p y)<\delta_{0}$, there exists a geodesic triangle $\widetilde{\triangle}(p x y):=\triangle(\tilde{p} \tilde{x} \tilde{y})$ in $\widetilde{V}\left(\delta_{0}\right)$ such that

$$
d(\tilde{p}, \tilde{x})=d(p, x), \quad d(\tilde{p}, \tilde{y})=d(p, y), \quad d(\tilde{x}, \tilde{y})=d(x, y)
$$


and that

$$
\angle(x p y) \geq \angle(\tilde{x} \tilde{p} \tilde{y}), \quad \angle(p x y) \geq \angle(\tilde{p} \tilde{x} \tilde{y}), \quad \angle(p y x) \geq \angle(\tilde{p} \tilde{y} \tilde{x}) .
$$

Here we denote by $d(\cdot, \cdot \cdot)$ the distance function induced from the Riemannian structure of $M$, or $\widetilde{M}$, and by $\angle(p x y)$ the angle between the minimal geodesics from $x$ to $p$ and $y$ forming the triangle $\triangle(p x y)$.

The assumption on $\widetilde{V}\left(\delta_{0}\right)$ in our comparison theorem is automatically satisfied if we employ a von Mangoldt surface of revolution, or a Cartan-Hadamard surface of revolution (i.e., $G$ is non-positive on $\widetilde{M})$ as an $(\widetilde{M}, \tilde{p})$. Therefore, our comparison theorem contains the Itokawa-Machigashira-Shiohama comparison theorem above as a corollary. The proof of our comparison theorem has completely different techniques from those used in [CE, Chapter 2], Sa, Chapter IV], [A], and [MS] (see Section 4 for the proof).

Before stating our main theorem, we will mention a few related results for our theorem to clarify our aim: Abresch and Gromoll $\mathrm{AG}$. proved the finiteness of the topological type of a complete open $n$-dimensional Riemannian manifold $X$ with non-negative Ricci curvature outside the open distance $t_{0}$-ball around $p \in X$ for some constant $t_{0}>0$, however, admitting sectional curvature bounded from below by some negative constant everywhere, and furthermore admitting diameter growth of small order $o\left(t^{1 / n}\right)$ for some $p \in X$. Here, " $X$ has finite topological type" means that $X$ is homeomorphic to the interior of a compact manifold with boundary. Although their result and technique have influenced many articles (cf. Sormani's study [So]), it looks very restrictive to assume that a complete open Riemannian $n$-manifold admits diameter growth of small order $o\left(t^{1 / n}\right)$ if once you live in the world of the radial curvature geometry. The next example shows that the diameter growth condition is too restrictive.

Example 1.1. Let $(\widetilde{M}, \tilde{p})$ be a non-negatively curved non-compact model surface of revolution with its metric (1.1), and admitting diameter growth $\mathcal{D}(t, \tilde{p})=o\left(t^{1 / 2}\right)$. We denote by $\mathcal{L}(t, \tilde{p})$ the length of the boundary of the open distance $t$-ball around $\tilde{p}$. Since $\mathcal{D}(t, \tilde{p})=o\left(t^{1 / 2}\right)$ and

$$
\lim _{t \rightarrow \infty} \frac{\mathcal{D}(t, \tilde{p})}{\mathcal{L}(t, \tilde{p})}
$$

is positive by [SST, Lemma 7.3.3], there exist positive numbers $\varepsilon_{1}$ and $\varepsilon_{2}$ such that

$$
2 \pi f(t)=\mathcal{L}(t, \tilde{p})=\frac{\mathcal{L}(t, \tilde{p})}{\mathcal{D}(t, \tilde{p})} \cdot \mathcal{D}(t, \tilde{p}) \leq \varepsilon_{1} \mathcal{D}(t, \tilde{p}) \leq \varepsilon_{2} \sqrt{t} .
$$

By (1.4), $\widetilde{M}$ satisfies

$$
\int_{1}^{\infty} \frac{1}{f(t)^{2}} d t=\infty
$$

Let $(M, p)$ be a complete open $n$-dimensional Riemannian manifold $M$ whose radial curvature at the base point $p$ is bounded from below by that of $\widetilde{M}$. Then (1.5) controls the topology of $(M, p)$; that is, it follows from [ST2, Theorem 1.2] that $(M, p)$ is isometric to the $n$-dimensional model $\widetilde{M}^{n}$ satisfying (1.5).

The authors [KT1 have recently reached a stronger conclusion than the Abresch and Gromoll result above, in which the diameter growth condition is replaced by an assumption on total curvatures of model surfaces. 
Theorem 1.2 ([KT1, Corollary to Main Theorem]). Let $(M, p)$ be a complete open Riemannian n-manifold $M$ whose radial curvature at the base point $p \in M$ is bounded from below by that of a non-compact von Mangoldt surface of revolution $(\widetilde{M}, \tilde{p})$. If $\widetilde{M}$ admits $c(\widetilde{M})>\pi$, then $M$ has finite topological type, and the isometry group of $M$ is compact. Here $c(\widetilde{M})$ denotes the total curvature of $\widetilde{M}$.

The assumption $c(\widetilde{M})>\pi$, of which one may complain of its being too strong, has been assumed in order to generalize Shiohama's result ([Sh1, Main Theorem]) in the geometry of complete open surfaces to any dimensional complete open Riemannian manifolds (see [KT1, Main Theorem]). Therefore, our main purpose of this article is to show the finiteness of topological type of a complete open Riemannian manifold with a wider class of metrics than those described in Theorem 1.2 .

Our main theorem is the following:

Main Theorem (Theorem 2.2 in Section 2). Let $(M, p)$ be a complete open Riemannian n-manifold $M$ whose radial curvature at the base point $p$ is bounded from below by that of a non-compact model surface of revolution $(\widetilde{M}, \tilde{p})$. If $\widetilde{M}$ admits $c(\widetilde{M})>-\infty$ and has no pair of cut points in $\widetilde{V}\left(\delta_{0}\right)$ for some $\delta_{0} \in(0, \pi)$, then $M$ has finite topological type.

Remark that $\widetilde{M}$ admits $c(\widetilde{M})>-\infty$ if and only if

$$
\int_{\widetilde{M}}|G| d \widetilde{M}<\infty
$$

(see [SST, Definition 2.1.3] and the paragraph after the definition).

In the Main Theorem, the assumption on the existence of $\widetilde{V}\left(\delta_{0}\right)$ is necessary in order to establish a new type of Toponogov comparison theorem as stated above. Notice that the model surface of revolution $\widetilde{M}$ in our Main Theorem cannot always be replaced by a Cartan-Hadamard surface of revolution with finite total curvature which bounds the radial curvature of $M$ from below.

Next, we will mention the significance of finite total curvature in the Main Theorem. Consider a non-compact model surface of revolution $(\widetilde{M}, \tilde{p})$ which satisfies one of the following conditions for some constant $R_{0}>0$ :

(VM) The radial curvature function is non-increasing on $\left[R_{0}, \infty\right)$.

(CH) The radial curvature function is non-positive on $\left[R_{0}, \infty\right)$.

Then, the following theorem clarifies the real significance of finite total curvature and also the validity of our Main Theorem:

Sector Theorem (Theorem 3.4 in Section 3 ). Let $(\widetilde{M}, \tilde{p})$ be a non-compact model surface of revolution satisfying the (VM), or the (CH) for some $R_{0}>0$. If $\widetilde{M}$ admits $c(\widetilde{M})>-\infty$, then there exists a number $\delta_{0} \in(0, \pi)$ such that there is no pair of cut points in $\widetilde{V}\left(\delta_{0}\right)$.

From the radial curvature geometry's standpoint, we therefore feel that our Main Theorem has a more natural assumption than that on the Abresch and Gromoll result above. Another related result for the Main Theorem is Sormani's [So]. She has proved that if a complete open $n$-dimensional Riemannian manifold with nonnegative Ricci curvature everywhere admits some diameter growth condition, then the manifold has a finitely generated fundamental group. However, by the same reason above, the diameter growth condition on her result also looks very restrictive. 
As an application of the Main Theorem and the Sector Theorem, we will present a partial answer to Milnor's open conjecture. The conjecture is stated as follows:

Milnor's open conjecture (see the line right after [Mi, Theorem 1]). A complete open Riemannian manifold with non-negative Ricci curvature everywhere must have a finitely generated fundamental group.

Then, the assumption on the existence of a non-compact model surface of revolution is natural. In fact, any complete open $n$-dimensional Riemannian manifold has a non-compact model surface of revolution:

Model Lemma (Lemma 5.1] in Section5). Let $M$ be a complete open Riemannian $n$-manifold and $p \in M$ any fixed point. Then, there exists a locally Lipschitz function $G(t)$ on $[0, \infty)$ such that the radial curvature of $M$ at $p$ is bounded from below by that of the non-compact model surface of revolution with radial curvature function $G(t)$.

Now, let $G(t)$ be the Lipschitz function in the Model Lemma, and set

$$
G^{*}(t):=\min \{0, G(t)\} .
$$

Consider a non-compact model surface of revolution $\left(M^{*}, p^{*}\right)$ with its metric

$$
g^{*}=d t^{2}+m(t)^{2} d \theta^{2}, \quad(t, \theta) \in(0, \infty) \times \mathbb{S}_{p^{*}}^{1}
$$

satisfying the differential equation

$$
m^{\prime \prime}(t)+G^{*}(t) m(t)=0
$$

with initial conditions $m(0)=0$ and $m^{\prime}(0)=1$. Notice that the metric (1.6) is not always differentiable around the base point $p^{*} \in M^{*}$. Then, it follows from the Model Lemma, the Sector Theorem, and the Main Theorem that we have the following partial answer to Milnor's open conjecture:

Partial answer to Milnor's open conjecture (Theorem5.3 in Section 5et $M$ be a complete open Riemannian $n$-manifold, $p \in M$ any fixed point, and $\left(M^{*}, p^{*}\right)$ a comparison model surface of revolution, constructed from $(M, p)$, with its metric (1.6). If $G^{*}(t)$ satisfies

$$
\int_{0}^{\infty}\left(-t \cdot G^{*}(t)\right) d t<\infty
$$

then the total curvature $c\left(M^{*}\right)$ is finite. In particular, then $M$ has a finitely generated fundamental group.

Another approach to Milnor's open conjecture has been done by Wilking [W]. He tried to prove the conjecture for manifolds with abelian fundamental groups; i.e., he proved that: Let $X$ be a complete $n$-dimensional Riemannian manifold $X$ with non-negative Ricci curvature everywhere, and $\widehat{X}$ the universal covering space of $X$. Then, if $I(\widehat{X}) / I_{0}(\widehat{X})$ is finitely generated, the fundamental group of $X$ is too. Here, $I_{0}(\widehat{X})$ denotes the identity component of the isometry group $I(\widehat{X})$ of $\widehat{X}$.

The organization of this article is as follows. In Section 2, this article reaches the climax; that is, we prove the Main Theorem (Theorem 2.2) by applying some results in Sections 3 and 4. In Section 3 , we investigate the relationship between a non-compact model surface of revolution $(\widetilde{M}, \tilde{p})$ with its finite total curvature and its cut locus. As a main theorem in Section 3, we finally prove the Sector Theorem (Theorem 3.4) using results in Section 3. In Section 4, we establish a new type of 
the Toponogov comparison theorem (Theorem 4.12). The key tools of the proof are our Alexandrov convexity (Lemma 4.4) and Essential Lemma (Lemma 4.11). In Section 5, we prove the Model Lemma (Lemma 5.1). After recalling some differential inequality (Lemma 5.2), we prove a partial answer (Theorem 5.3) to Milnor's open conjecture, using the Model Lemma, the differential inequality, the Sector Theorem, and the Main Theorem. Finally, we prove a corollary (Corollary 5.4) to the Main Theorem, which is another partial answer to Milnor's open conjecture.

In the following sections, all geodesics will be normalized, unless otherwise stated.

\section{Proof of the Main Theorem}

In 1977, Grove and Shiohama GS first introduced the notion of a critical point of distance functions to prove their famous theorem, which is called the diameter sphere theorem. The definition of the critical point of distance functions is given as follows:

Definition 2.1. Let $M$ be a complete Riemannian manifold. For any fixed point $p \in M$, a point $q \in M \backslash\{p\}$ is called a critical point of $d(p, \cdot)$ (or critical point for $p$ ) if, for every non-zero tangent vector $v \in T_{q} M$, we find a minimal geodesic $\gamma$ emanating from $q$ to $p$ satisfying

$$
\angle\left(v, \gamma^{\prime}(0)\right) \leq \frac{\pi}{2}
$$

Here, we denote by $\angle\left(v, \gamma^{\prime}(0)\right)$ the angle between two vectors $v$ and $\gamma^{\prime}(0)$ in $T_{q} M$.

In 1981, Gromov G1 refined the technique given by Grove and Shiohama in GS in order to estimate an upper bound on the sum of the Betti numbers over any fixed field for compact (connected) Riemannian manifolds with non-negative sectional curvature everywhere:

Gromov's Isotopy Lemma. Let $M$ be a complete Riemannian manifold. If $0<R_{1}<R_{2} \leq \infty$, and if $\overline{B_{R_{2}}(p)} \backslash B_{R_{1}}(p)$ has no critical point for $p \in M$, then $\overline{B_{R_{2}}(p)} \backslash B_{R_{1}}(p)$ is homeomorphic to $\partial B_{R_{1}}(p) \times\left[R_{1}, R_{2}\right]$. Here, $B_{R_{i}}(p), i=1,2$, are the open distance $R_{i}$-balls around $p$.

From now on, let $(\widetilde{M}, \tilde{p})$ be a non-compact model surface of revolution, and let $(M, p)$ be a complete open Riemannian $n$-manifold $M$ whose radial curvature at the base point $p \in M$ is bounded from below by that of the $(\widetilde{M}, \tilde{p})$. Admitting Lemmas 3.2, 4.11, and Theorem 4.12 in Sections 3 and 4, we can prove that:

Theorem 2.2. If $\widetilde{M}$ admits $c(\widetilde{M})>-\infty$ and has no pair of cut points in $\widetilde{V}\left(\delta_{0}\right)$ for some $\delta_{0} \in(0, \pi)$, then $M$ has finite topological type.

Proof. From the Isotopy Lemma, it is sufficient to prove that the set of critical points of $d(p, \cdot)$ is bounded. Suppose that there exists a divergent sequence $\left\{q_{i}\right\}$ of critical points $q_{i} \in M$ of $d(p, \cdot)$. Let $\gamma_{i}:\left[0, d\left(p, q_{i}\right)\right] \longrightarrow M$ be a minimal geodesic segment emanating from $p$ to $q_{i}$. We may assume, by taking a subsequence of $\left\{q_{i}\right\}$, if necessary, that the sequence $\left\{\gamma_{i}\right\}$ converges to a ray $\gamma$ emanating from $p$. Thus, there exists a sufficiently large $i_{0} \in \mathbb{N}$ such that

$$
\angle\left(\gamma^{\prime}(0), \gamma_{i}^{\prime}(0)\right)<\delta_{0}
$$

for any $i \geq i_{0}$. From now on, choose any $i \geq i_{0}$ and fix it. Let $\varepsilon$ be an arbitrary positive number less than $\pi / 2$. Applying the Cohn-Vossen technique (see [CV], or 
[SST, Lemma 2.2.1]), we can choose a positive number $t_{i}$ satisfying

$$
\angle\left(\gamma^{\prime}\left(t_{i}\right), \eta_{i}^{\prime}\left(s_{i}\right)\right)<\varepsilon \text {. }
$$

Here, $\eta_{i}:\left[0, s_{i}\right] \longrightarrow M$ denotes a minimal geodesic segment emanating from $q_{i}$ to $\gamma\left(t_{i}\right)$. Since the geodesic triangle $\triangle\left(p q_{i} \gamma\left(t_{i}\right)\right) \subset M$, consisting of the edges $\gamma_{i}, \eta_{i}$, and $\left.\gamma\right|_{\left[0, t_{i}\right]}$, satisfies

$$
\angle\left(q_{i} p \gamma\left(t_{i}\right)\right)<\delta_{0}
$$

by (2.1), it follows from Theorem 4.12 and (2.2) that there exists a geodesic triangle $\widetilde{\triangle}\left(p q_{i} \gamma\left(t_{i}\right)\right):=\triangle\left(\tilde{p} \tilde{q}_{i} \tilde{\gamma}\left(t_{i}\right)\right) \subset \widetilde{M}$ satisfying (4.53) (for $x=q_{i}$ and $y=\gamma\left(t_{i}\right)$ ),

$$
\angle\left(q_{i} p \gamma\left(t_{i}\right)\right) \geq \angle\left(\tilde{q}_{i} \tilde{p} \tilde{\gamma}\left(t_{i}\right)\right)
$$

and

$$
\angle\left(\tilde{q}_{i} \tilde{\gamma}\left(t_{i}\right) \tilde{p}\right)<\varepsilon
$$

Since

$$
\lim _{i \rightarrow \infty} \angle\left(q_{i} p \gamma\left(t_{i}\right)\right)=0
$$

we have

$$
\lim _{i \rightarrow \infty} \angle\left(\tilde{q}_{i} \tilde{p} \tilde{\gamma}\left(t_{i}\right)\right)=0 .
$$

On the other hand, since each $q_{i}$ is a critical point of $d(p, \cdot)$, there exists a minimal geodesic segment $\sigma_{i}:\left[0, d\left(p, q_{i}\right)\right] \longrightarrow M$ emanating from $q_{i}$ to $p$ such that

$$
\angle\left(\sigma_{i}^{\prime}(0), \eta_{i}^{\prime}(0)\right) \leq \frac{\pi}{2}
$$

Let $\triangle\left(p \sigma_{i}(0) \gamma\left(t_{i}\right)\right) \subset M$ denote the geodesic triangle consisting of the edges $\sigma_{i}$, $\eta_{i}$, and $\left.\gamma\right|_{\left[0, t_{i}\right]}$. Since $\triangle\left(p \sigma_{i}(0) \gamma\left(t_{i}\right)\right)$ has the same side lengths as $\triangle\left(p q_{i} \gamma\left(t_{i}\right)\right)$, the triangle $\triangle\left(p \sigma_{i}(0) \gamma\left(t_{i}\right)\right)$ admits the triangle $\widetilde{\triangle}\left(p q_{i} \gamma\left(t_{i}\right)\right) \subset \widetilde{V}\left(\delta_{0}\right)$ satisfying (4.51) (for $x=\sigma_{i}(0)$ and $y=\gamma\left(t_{i}\right)$ ) in Lemma 4.11. Thus, by Lemma 4.11, we have

$$
\angle\left(\sigma_{i}^{\prime}(0), \eta_{i}^{\prime}(0)\right) \geq \angle\left(\tilde{p} \tilde{q}_{i} \tilde{\gamma}\left(t_{i}\right)\right) .
$$

By (2.5) and (2.6), we get

$$
\angle\left(\tilde{p} \tilde{q}_{i} \tilde{\gamma}\left(t_{i}\right)\right) \leq \frac{\pi}{2}
$$

Applying the Gauss - Bonnet Theorem to the geodesic triangle $\widetilde{\triangle}\left(p q_{i} \gamma\left(t_{i}\right)\right)$, we have

$$
\int_{\widetilde{\triangle}\left(p q_{i} \gamma\left(t_{i}\right)\right)} G d \widetilde{M}=\angle\left(\tilde{q}_{i} \tilde{p} \tilde{\gamma}\left(t_{i}\right)\right)+\angle\left(\tilde{p} \tilde{q}_{i} \tilde{\gamma}\left(t_{i}\right)\right)+\angle\left(\tilde{q}_{i} \tilde{\gamma}\left(t_{i}\right) \tilde{p}\right)-\pi .
$$

Since

$$
\lim _{i \rightarrow \infty} \int_{\widetilde{\triangle}\left(p q_{i} \gamma\left(t_{i}\right)\right)} G d \widetilde{M}=0
$$

by Lemma 3.2, we have, by (2.8),

$$
\lim _{i \rightarrow \infty}\left(\angle\left(\tilde{q}_{i} \tilde{p} \tilde{\gamma}\left(t_{i}\right)\right)+\angle\left(\tilde{p} \tilde{q}_{i} \tilde{\gamma}\left(t_{i}\right)\right)+\angle\left(\tilde{q}_{i} \tilde{\gamma}\left(t_{i}\right) \tilde{p}\right)\right)=\pi .
$$

By (2.3), (2.4), (2.7), and (2.9), we get

$$
\frac{\pi}{2} \leq \varepsilon .
$$

This is a contradiction. Therefore, the set of critical points of $d(p, \cdot)$ is bounded. 


\section{Sector TheOrem AND CUt LOCI OF MODEL SURFACES}

We will first introduce some fundamental tools in the geometry of surfaces of revolution. For details of the geometry, readers can refer to [SST, Chapter 7] (also refer to [T1], GMST], and [SiT2]). Observe that the following results hold for all non-compact model surfaces of revolution except for Theorem 3.4.

For a non-compact model surface of revolution $(\widetilde{M}, \tilde{p})$ whose metric satisfies (1.1), a unit speed geodesic $\tilde{\sigma}:[0, a) \longrightarrow \widetilde{M}(0<a \leq \infty)$ is expressed by

$$
\tilde{\sigma}(s)=(t(\tilde{\sigma}(s)), \theta(\tilde{\sigma}(s)))=:(t(s), \theta(s)) .
$$

Then, there exists a non-negative constant $\nu$ depending only on $\tilde{\sigma}$ such that

$$
\nu=f(t(s))^{2}\left|\theta^{\prime}(s)\right|=f(t(s)) \sin \angle\left(\tilde{\sigma}^{\prime}(s),(\partial / \partial t)_{\tilde{\sigma}(s)}\right) .
$$

This (3.1) is a famous formula, which is called the Clairaut relation. The constant $\nu$ is called the Clairaut constant of $\tilde{\sigma}$. Notice that, by (3.1),

$$
\nu>0 \text { if and only if } \tilde{\sigma} \text { is not a meridian, or its subarc. }
$$

Since $\tilde{\sigma}$ is unit speed, we have, by (3.1),

$$
t^{\prime}(s)= \pm \frac{\sqrt{f(t(s))^{2}-\nu^{2}}}{f(t(s))} .
$$

Observe that, by (3.2),

$$
t^{\prime}(s)=0 \text { if and only if } f(t(s))=\nu .
$$

It follows from (3.1) and (3.2) that, for a unit speed geodesic $\tilde{\sigma}(s)=(t(s), \theta(s))$, $s_{1} \leq s \leq s_{2}$, with the Clairaut constant $\nu$,

$$
\theta\left(s_{2}\right)-\theta\left(s_{1}\right)=\lambda\left(t^{\prime}(s)\right) \int_{t\left(s_{1}\right)}^{t\left(s_{2}\right)} \frac{\nu}{f(t) \sqrt{f(t)^{2}-\nu^{2}}} d t
$$

holds if $t^{\prime}(s) \neq 0$ on $\left(s_{1}, s_{2}\right)$. Here, $\lambda\left(t^{\prime}(s)\right)$ denotes the sign of $t^{\prime}(s)$.

Lemma 3.1. Let $(\widetilde{M}, \tilde{p})$ be a non-compact model surface of revolution, and $\widetilde{V}_{i}$ denote $\widetilde{V}(1 / i)$ for each $i \in \mathbb{N}$. Assume that there exist a constant $t_{0}>0$ and a sequence

$$
\left\{\tilde{\sigma}_{i}:\left[0, \ell_{i}\right] \longrightarrow \widetilde{V}_{i}\right\}_{i \in \mathbb{N}}
$$

of unit speed geodesic segments such that

$$
\tilde{\sigma}_{i}\left(\left[0, \ell_{i}\right]\right) \cap \overline{B_{t_{0}}(\tilde{p})} \neq \emptyset
$$

for each $i \in \mathbb{N}$, and that

$$
\liminf _{i \rightarrow \infty} t\left(\tilde{\sigma}_{i}\left(\ell_{i}\right)\right)>t_{0}
$$

Then,

$$
\lim _{i \rightarrow \infty} \nu_{i}=0
$$

holds. Here, $\nu_{i}$ denotes the Clairaut constant of $\tilde{\sigma}_{i}$.

Proof. By the assumptions (3.4) and (3.5), we can find a constant number

$$
u_{i}:=\sup \left\{s \in\left(0, \ell_{i}\right) \mid t\left(\tilde{\sigma}_{i}(s)\right)=t_{0}\right\}
$$

for each $i \in \mathbb{N}$. Moreover, it follows from (3.5) that for any sufficiently large $i \in \mathbb{N}$, there exists a constant number $t_{1}>0$ such that

$$
t_{0}<t_{1}<t\left(\tilde{\sigma}_{i}\left(\ell_{i}\right)\right)
$$


Then, we can find a constant number $v_{i} \in\left(t \circ \tilde{\sigma}_{i}\right)^{-1}\left(t_{1}\right) \subset\left(0, \ell_{i}\right)$ such that $v_{i}>u_{i}$. Since $\tilde{\sigma}_{i}\left(\left[u_{i}, v_{i}\right]\right) \subset \widetilde{V}_{i}$, we have, by (3.3),

$$
\begin{aligned}
\frac{1}{i} & >\int_{t\left(\tilde{\sigma}_{i}\left(u_{i}\right)\right)}^{t\left(\tilde{\sigma}_{i}\left(v_{i}\right)\right)} \frac{\nu_{i}}{f(t) \sqrt{f(t)^{2}-\nu_{i}^{2}}} d t \\
& =\int_{t_{0}}^{t_{1}} \frac{\nu_{i}}{f(t) \sqrt{f(t)^{2}-\nu_{i}^{2}}} d t \\
& \geq \nu_{i} \cdot \int_{t_{0}}^{t_{1}} \frac{1}{f(t)^{2}} d t>0
\end{aligned}
$$

for any sufficiently large $i \in \mathbb{N}$. Thus, by (3.6), we get

$$
\lim _{i \rightarrow \infty} \nu_{i}=0 \text {. }
$$

Lemma 3.2. Let $(\widetilde{M}, \tilde{p})$ be a non-compact model surface of revolution, and set

$$
\bar{V}\left(\theta_{i}\right):=\left\{\tilde{x} \in \widetilde{M} \mid 0 \leq \theta(\tilde{x}) \leq \theta_{i}\right\}
$$

for each $i \in \mathbb{N}$, where $\left\{\theta_{i}\right\}$ is a sequence of positive numbers convergent to 0 . If $\widetilde{M}$ admits $c(\widetilde{M})>-\infty$, then

$$
\lim _{i \rightarrow \infty} \int_{\bar{V}\left(\theta_{i}\right)}|G| d \widetilde{M}=0
$$

holds. In particular,

$$
\lim _{i \rightarrow \infty} \int_{\widetilde{\triangle}_{i}} G d \widetilde{M}=0
$$

holds for any sequence $\left\{\widetilde{\triangle}_{i}\right\}$ of geodesic triangles $\widetilde{\triangle}_{i} \subset \bar{V}\left(\theta_{i}\right)$.

Proof. Since $c(\widetilde{M})>-\infty$, for any $\varepsilon>0$, there exists a number $r(\varepsilon)>0$ such that

$$
\int_{\widetilde{M} \backslash B_{r(\varepsilon)}(\tilde{p})}|G| d \widetilde{M}<\frac{\varepsilon}{2}
$$

where $B_{r(\varepsilon)}(\tilde{p}) \subset \widetilde{M}$ is the open distance $r(\varepsilon)$-ball around $\tilde{p} \in \widetilde{M}$. Then, there exists $i_{0}(\varepsilon) \in \mathbb{N}$ such that

$$
\int_{\bar{V}\left(\theta_{i}\right) \cap B_{r(\varepsilon)}(\tilde{p})}|G| d \widetilde{M}=\frac{\theta_{i}}{2 \pi} \int_{B_{r(\varepsilon)}(\tilde{p})}|G| d \widetilde{M}<\frac{\varepsilon}{2}
$$

holds for all $i>i_{0}(\varepsilon)$. Therefore, by (3.7) and (3.8), we get the first assertion, that is,

$$
\int_{\bar{V}\left(\theta_{i}\right)}|G| d \widetilde{M} \leq \int_{\bar{V}\left(\theta_{i}\right) \cap B_{r(\varepsilon)}(\tilde{p})}|G| d \widetilde{M}+\int_{\widetilde{M} \backslash B_{r(\varepsilon)}(\tilde{p})}|G| d \widetilde{M}<\varepsilon
$$

for all $i>i_{0}(\varepsilon)$. Furthermore, by (3.9),

$$
\left|\int_{\widetilde{\triangle}_{i}} G d \widetilde{M}\right| \leq \int_{\widetilde{\triangle}_{i}}|G| d \widetilde{M} \leq \int_{\bar{V}\left(\theta_{i}\right)}|G| d \widetilde{M}<\varepsilon
$$

holds for all $i>i_{0}(\varepsilon)$, which is the second assertion. 
Lemma 3.3 (Key Lemma). Let $(\widetilde{M}, \tilde{p})$ be a non-compact model surface of revolution. If $\widetilde{M}$ admits $c(\widetilde{M})>-\infty$, then, for each $t>0$, there exists a constant number $\delta(t) \in(0, \pi)$ such that

$$
\tilde{\sigma}([0, \ell]) \cap \overline{B_{t}(\tilde{p})}=\emptyset
$$

holds for any minimal geodesic segment $\tilde{\sigma}:[0, \ell] \longrightarrow \widetilde{V}(\delta(t)) \subset \widetilde{M}$, along which $\tilde{\sigma}(0)$ is conjugate to $\tilde{\sigma}(\ell)$.

Proof. Since $|\theta(\tilde{\sigma}(0))-\theta(\tilde{\sigma}(\ell))|<\pi$ holds for all minimal geodesic segments $\tilde{\sigma}$ : $[0, \ell] \longrightarrow \widetilde{M} \backslash\{\tilde{p}\}$, it is sufficient to show that a required number $\delta(t)$ is positive. Suppose that there exist a constant $t_{0}>0$ and a sequence

$$
\left\{\tilde{\sigma}_{i}:\left[0, \ell_{i}\right] \longrightarrow \widetilde{V}_{i}\right\}_{i \in \mathbb{N}}
$$

of minimal geodesic segments, along which $\tilde{\sigma}_{i}(0)$ is conjugate to $\tilde{\sigma}_{i}\left(\ell_{i}\right)$ for each $i \in \mathbb{N}$, such that

$$
\tilde{\sigma}_{i}\left(\left[0, \ell_{i}\right]\right) \cap \overline{B_{t_{0}}(\tilde{p})} \neq \emptyset
$$

for each $i \in \mathbb{N}$. Here, we set $\widetilde{V}_{i}:=\widetilde{V}(1 / i)$ for each $i \in \mathbb{N}$. Since $\tilde{\sigma}_{i}(0)$ is conjugate to $\tilde{\sigma}_{i}\left(\ell_{i}\right)$ along $\tilde{\sigma}_{i}$ for each $i \in \mathbb{N}$, we see that $\lim _{i \rightarrow \infty} \ell_{i}=\infty$, and hence we may assume that

$$
\liminf _{i \rightarrow \infty} t\left(\tilde{\sigma}_{i}\left(\ell_{i}\right)\right)>t_{0} .
$$

Thus, by Lemma 3.1] we have

$$
\lim _{i \rightarrow \infty} \nu_{i}=0,
$$

where $\nu_{i}$ is the Clairaut constant of $\tilde{\sigma}_{i}$. Since $\tilde{\sigma}_{i}(0)$ is conjugate to $\tilde{\sigma}_{i}\left(\ell_{i}\right)$ along $\tilde{\sigma}_{i}$ for each $i \in \mathbb{N}$, there exists $a_{i} \in\left[0, \ell_{i}\right]$ such that

$$
\left(t \circ \tilde{\sigma}_{i}\right)^{\prime}\left(a_{i}\right)=0
$$

(cf. [SST, Proposition 7.2.1]). Let $u_{i} \in\left[0, \ell_{i}\right]$ be the parameter value of $\tilde{\sigma}_{i}$ such that

$$
t\left(\tilde{\sigma}_{i}\left(u_{i}\right)\right)=t_{0} .
$$

We consider a geodesic triangle $\widetilde{\triangle}_{i}:=\triangle\left(\tilde{p} \tilde{\sigma}_{i}\left(a_{i}\right) \tilde{\sigma}_{i}\left(u_{i}\right)\right)$ in $\widetilde{V}_{i}$. It follows from (3.1) and (3.12) that

$$
\lim _{i \rightarrow \infty} \sin \left(\angle\left(\tilde{p} \tilde{\sigma}_{i}\left(u_{i}\right) \tilde{\sigma}_{i}\left(a_{i}\right)\right)\right)=\lim _{i \rightarrow \infty} \frac{\nu_{i}}{f\left(t_{0}\right)}=0
$$

holds. Furthermore, by (3.13), we have

$$
\angle\left(\tilde{p} \tilde{\sigma}_{i}\left(a_{i}\right) \tilde{\sigma}_{i}\left(u_{i}\right)\right)=\frac{\pi}{2} .
$$

Observe that

$$
\lim _{i \rightarrow \infty} \angle\left(\tilde{\sigma}_{i}\left(a_{i}\right) \tilde{p} \tilde{\sigma}_{i}\left(u_{i}\right)\right)=0,
$$

since $\widetilde{\triangle}_{i} \subset \widetilde{V}_{i}$ for each $i \in \mathbb{N}$. By Lemma 3.2, (3.15), (3.16), and the Gauss - Bonnet Theorem, we get

$$
\lim _{i \rightarrow \infty} \angle\left(\tilde{p} \tilde{\sigma}_{i}\left(u_{i}\right) \tilde{\sigma}_{i}\left(a_{i}\right)\right)=\frac{\pi}{2} .
$$

The equation (3.17) contradicts the equation (3.14). 
Hebda [He proved that the cut locus $\operatorname{Cut}(x)$ of a point $x$ in a complete Riemannian 2-manifold is a local tree; that is, for any $y \in \operatorname{Cut}(x)$ and any neighborhood $\mathcal{U}$ around $y$ in the surface, there exists an open neighborhood $\mathcal{O} \subset \mathcal{U}$ around $y$ such that any two cut points in $\mathcal{O}$ can be joined by a unique rectifiable Jordan arc in $\mathcal{O} \cap \operatorname{Cut}(x)$ (see also ST1 for Alexandrov surfaces). Here, a Jordan arc means an arc homeomorphic to the interval $[0,1]$.

Theorem 3.4 (Sector Theorem). Let $(\widetilde{M}, \tilde{p})$ be a non-compact model surface of revolution satisfying the (VM), or the $(C H)$ for some $R_{0}>0$. If $\widetilde{M}$ admits $c(\widetilde{M})>$ $-\infty$, then there exists a positive number $\delta_{0} \in(0, \pi)$ such that $\widetilde{V}\left(\delta_{0}\right)$ has no pair of cut points.

Proof. Choose any number $R_{1}>R_{0}$ and fix it. We will prove that $\widetilde{V}\left(\delta\left(R_{1}\right)\right)$ has no pair of cut points, where $\delta\left(R_{1}\right) \in(0, \pi)$ is the number guaranteed in Lemma 3.3. Suppose that $\widetilde{V}\left(\delta\left(R_{1}\right)\right)$ has a pair of cut points $\tilde{x}$ and $\tilde{y}$. Let $\tilde{\sigma}:[0, d(\tilde{x}, \tilde{y})] \longrightarrow$ $\widetilde{V}\left(\delta\left(R_{1}\right)\right)$ denote a minimal geodesic segment joining $\tilde{x}$ to $\tilde{y}$. We may assume that $\tilde{x}$ is conjugate to $\tilde{y}$ along $\tilde{\sigma}$. Otherwise, we may find another minimal geodesic segment $\tilde{\alpha}$ joining $\tilde{x}$ to $\tilde{y}$. Clearly $\tilde{\sigma}$ and $\tilde{\alpha}$ bound a relatively compact domain $\mathcal{D}$ in $\widetilde{V}\left(\delta\left(R_{1}\right)\right)$. Since $\operatorname{Cut}(\tilde{x})$ is a tree, we may find an endpoint $\tilde{z} \in \mathcal{D}$ of $\operatorname{Cut}(\tilde{x})$. Then, $\tilde{x}$ is conjugate to $\tilde{z}$ along any minimal geodesic segment joining $\tilde{x}$ to $\tilde{z}$. By exchanging $\tilde{y}$ and $\tilde{z}$, we may assume that $\tilde{x}$ is conjugate to $\tilde{y}$ along $\tilde{\sigma}$. From Lemma 3.3. the minimal geodesic segment $\tilde{\sigma}$ does not intersect $\overline{B_{R_{1}}(\tilde{p})}$. If $G \circ \tilde{\gamma}\left(R_{1}\right)$ is non-positive, then $G \circ \tilde{\gamma}(t) \leq 0$ holds on $\left[R_{1}, \infty\right)$, where $\tilde{\gamma}$ denotes any meridian emanating from $\tilde{p}=\tilde{\gamma}(0)$. Hence $G(\tilde{\sigma}(s)) \leq 0$ holds for all $s \in[0, d(\tilde{x}, \tilde{y})]$. This contradicts the property that $\tilde{x}$ is conjugate to $\tilde{y}$ along $\tilde{\sigma}$. If $G \circ \tilde{\gamma}\left(R_{1}\right)$ is positive and $G \circ \tilde{\gamma}$ is non-increasing on $\left[R_{0}, \infty\right)$, where $\tilde{\gamma}$ denotes any meridian emanating from $\tilde{p}=\tilde{\gamma}(0)$, then since $\tilde{y}$ is not a single cut point of $\tilde{x}$, we may find a unit speed, rectifiable Jordan $\operatorname{arc} \tilde{\xi}(r)$ in $\operatorname{Cut}(\tilde{x})$ emanating from $\tilde{y}=\tilde{\xi}(0)$. Since $\tilde{\sigma}$ does not intersect $\overline{B_{R_{1}}(\tilde{p})}$, there exists a sufficiently small number $\varepsilon_{0}>0$ such that the domain bounded by two minimal geodesic segments $\tilde{\tau}$ and $\tilde{\eta}$ joining $\tilde{x}$ to $\tilde{\xi}\left(\varepsilon_{0}\right)$ does not intersect $\overline{B_{R_{1}}(\tilde{p})}$. We assume that $\tilde{\tau}$ and $\tilde{\eta}$ are chosen in such a way that

$$
\angle\left(\tilde{\eta}^{\prime}(0),(\partial / \partial t)_{\tilde{x}}\right)<\angle\left(\tilde{\sigma}^{\prime}(0),(\partial / \partial t)_{\tilde{x}}\right)<\angle\left(\tilde{\tau}^{\prime}(0),(\partial / \partial t)_{\tilde{x}}\right) .
$$

Then, we may get a contradiction by repeating the argument in the proof of GMST, Lemma 3.1] or [SiT2, Lemma 3.1]. In the following, we hence state only the sketch of the argument. We may prove that $\tilde{\sigma}$ is shorter than $\tilde{\tau}$, and that

$$
t(\tilde{\sigma}(s)) \geq t(\tilde{\tau}(s))
$$

for all $s \in[0, d(\tilde{x}, \tilde{y})]$. The equation (3.18) implies that

$$
G(\tilde{\sigma}(s)) \leq G(\tilde{\tau}(s))
$$

for all $s \in[0, d(\tilde{x}, \tilde{y})]$, since $G \circ \tilde{\gamma}$ is non-increasing on $\left[R_{0}, \infty\right)$. From the Rauch comparison theorem and (3.19), the geodesic segment $\left.\tilde{\tau}\right|_{[0, d(\tilde{x}, \tilde{y})]}$ has a conjugate point of $\tilde{x}$ along the segment. This contradicts the fact that $\tilde{\tau}$ is minimal. Therefore, $\widetilde{V}\left(\delta\left(R_{1}\right)\right)$ has no pair of cut points.

\section{A NeW TYPe OF the TOPONOGOV COMPARISON THEOREM}

In the pure sectional curvature geometry, the Toponogov comparison theorem has been a very important tool in the investigation of the relationship between 
the curvature and topology of Riemannian manifolds (cf. [B], CG], GS], G1], and so on). After the Gromov convergence theorem [G2] and the Grove and Peterson finiteness theorem for homotopy and diffeomorphism types [GP, GPW], it has become very important to investigate the topology of Alexandrov spaces as one of the terminal stations of the pure sectional curvature geometry (cf. [Al], BGP, [P1, [P2, O1, O2], OS], ST1, SY1, [SY2, [Y], and so on).

As stated in Section 1, such comparison theorems in the radial curvature geometry were proved by making use of model surfaces of revolution instead of complete surfaces of constant Gaussian curvature (see [A], [MS], and [SiT2]). In the radial curvature geometry, all geodesic triangles must have the base point as one of the vertices. Thus, the radial curvature geometry looks more restricted than the pure sectional curvature geometry, but this is not the case. We should remark that we can construct a model surface of revolution for any complete Riemannian manifold with an arbitrary given point as a base point (see Model Lemma in Subsection 5.1).

Our purpose in this section is to establish a new type of Toponogov comparison theorem (Theorem 4.12). The key tools of the proof are our Alexandrov convexity (Lemma 4.4) and the Essential Lemma (Lemma 4.11).

4.1. Alexandrov convexity. The Alexandrov convexity at a base point was proved in IMS when comparison surfaces are von Mangoldt surfaces of revolution. We first establish our Alexandrov convexity (Lemma 4.4) at a base point in a more general situation. For this purpose, we need three lemmas (Lemmas 4.14 .2 . and 4.3 ).

Let $(\widetilde{M}, \tilde{p})$ be an arbitrary non-compact model surface of revolution whose metric satisfies (1.1). Then, we have the next lemma, which is a very important tool in the investigation of radial curvature geometry.

Lemma 4.1 ([SST, Lemma 7.3.2]). Take a point $\tilde{q} \in \widetilde{M} \backslash\{\tilde{p}\}$ with $\theta(\tilde{q})=0$. If two points $\tilde{x}_{1}, \tilde{x}_{2} \in \widetilde{M}$ satisfy $t\left(\tilde{x}_{1}\right)=t\left(\tilde{x}_{2}\right)$ and $0 \leq \theta\left(\tilde{x}_{1}\right)<\theta\left(\tilde{x}_{2}\right) \leq \pi$, then,

$$
d\left(\tilde{q}, \tilde{x}_{1}\right)<d\left(\tilde{q}, \tilde{x}_{2}\right)
$$

holds.

Let $\tilde{\mu}_{\alpha}:[0, \infty) \longrightarrow \widetilde{M}$ denote the meridian defined by $\theta=\alpha$, which emanates from $\tilde{p}$. It follows from Lemma 4.1 that, for any $a>0, c>0$, and $\theta_{0} \in(0, \pi)$,

$$
\liminf _{\theta \downarrow \theta_{0}} \frac{d\left(\tilde{\mu}_{0}(a), \tilde{\sigma}_{c}(\theta)\right)-d\left(\tilde{\mu}_{0}(a), \tilde{\sigma}_{c}\left(\theta_{0}\right)\right)}{\theta-\theta_{0}} \geq 0
$$

where $\tilde{\sigma}_{c}:[0,2 \pi) \longrightarrow \widetilde{M}$ denotes the parallel $t=c$, that is, $\tilde{\sigma}_{c}(\theta):=\tilde{\mu}_{\theta}(c)$. In the next lemma, we will prove that the left-hand term in the equation (4.1) is strictly positive.

Lemma 4.2. For any $a_{0}>0, c_{0}>0$, and $\theta_{0} \in(0, \pi)$, there exist constant numbers $\varepsilon_{1} \in(0, \pi / 2)$ and $\delta>0$ such that

$$
\left|d\left(\tilde{\mu}_{0}(a), \tilde{\sigma}_{c}\left(\theta_{2}\right)\right)-d\left(\tilde{\mu}_{0}(a), \tilde{\sigma}_{c}\left(\theta_{1}\right)\right)\right| \geq\left(f(c) \sin \varepsilon_{1}\right)\left|\theta_{2}-\theta_{1}\right|
$$

holds for all $a \in\left(a_{0}-\delta, a_{0}+\delta\right), c \in\left(c_{0}-\delta, c_{0}+\delta\right)$, and $\theta_{1}, \theta_{2} \in\left(\theta_{0}-\delta, \theta_{0}+\delta\right)$.

Proof. Choose any $a \in\left(a_{0}-\delta, a_{0}+\delta\right), c \in\left(c_{0}-\delta, c_{0}+\delta\right)$, and $\theta \in\left(\theta_{0}-\delta, \theta_{0}+\delta\right)$, where $\delta$ is a fixed positive number less than

$$
\frac{1}{3} \min \left\{a_{0}, \theta_{0}, c_{0}, \pi-\theta_{0}\right\} \text {. }
$$


Since no minimal geodesic segment joining $\tilde{\mu}_{0}(a)$ to $\tilde{\sigma}_{c}(\theta)$ is tangent to the meridian $\tilde{\mu}_{\theta}$, there exists a positive constant $\varepsilon_{1} \in(0, \pi / 2)$ such that

$$
\Phi(\tilde{\gamma}, \theta):=\angle\left(\tilde{\sigma}_{c}^{\prime}(\theta), \tilde{\gamma}^{\prime}\left(d\left(\tilde{\mu}_{0}(a), \tilde{\sigma}_{c}(\theta)\right)\right)\right) \leq \frac{\pi}{2}-\varepsilon_{1}
$$

holds for all $a \in\left(a_{0}-\delta, a_{0}+\delta\right), \theta \in\left(\theta_{0}-\delta, \theta_{0}+\delta\right)$, and all minimal geodesic segments $\tilde{\gamma}$ joining $\tilde{\mu}_{0}(a)$ to $\tilde{\sigma}_{c}(\theta)$. Therefore, it follows from [IT, Lemma 2.1] and (4.3) that, for each $\theta_{1} \in\left(\theta_{0}-\delta, \theta_{0}+\delta\right)$,

$$
\begin{aligned}
\liminf _{\theta \downarrow \theta_{1}} \frac{d\left(\tilde{\mu}_{0}(a), \tilde{\sigma}_{c}(\theta)\right)-d\left(\tilde{\mu}_{0}(a), \tilde{\sigma}_{c}\left(\theta_{1}\right)\right)}{d\left(\tilde{\sigma}_{c}(\theta), \tilde{\sigma}_{c}\left(\theta_{1}\right)\right)} & =-\cos \left(\min _{\tilde{\gamma}}\left\{\pi-\Phi\left(\tilde{\gamma}, \theta_{1}\right)\right\}\right) \\
& \geq-\cos \left(\frac{\pi}{2}+\varepsilon_{1}\right) \\
& =\sin \varepsilon_{1} .
\end{aligned}
$$

Since

$$
\lim _{\theta \rightarrow \theta_{1}} \frac{d\left(\tilde{\sigma}_{c}(\theta), \tilde{\sigma}_{c}\left(\theta_{1}\right)\right)}{\left|\theta-\theta_{1}\right|}=f(c),
$$

we get, by (4.4),

$$
\liminf _{\theta \downarrow \theta_{1}} \frac{d\left(\tilde{\mu}_{0}(a), \tilde{\sigma}_{c}(\theta)\right)-d\left(\tilde{\mu}_{0}(a), \tilde{\sigma}_{c}\left(\theta_{1}\right)\right)}{\theta-\theta_{1}} \geq f(c) \sin \varepsilon_{1} .
$$

Since $d\left(\tilde{\mu}_{0}(a), \tilde{\sigma}_{c}(\theta)\right)$ is Lipschitz with respect to $\theta$, it follows from Dini's theorem D] (cf. [Hw, Section 2.3], [WZ, Theorem 7.29]) that $d\left(\tilde{\mu}_{0}(a), \tilde{\sigma}_{c}(\theta)\right)$ is differentiable almost everywhere and

$$
\int_{\theta_{1}}^{\theta_{2}} \frac{\partial}{\partial \theta} d\left(\tilde{\mu}_{0}(a), \tilde{\sigma}_{c}(\theta)\right) d \theta=d\left(\tilde{\mu}_{0}(a), \tilde{\sigma}_{c}\left(\theta_{2}\right)\right)-d\left(\tilde{\mu}_{0}(a), \tilde{\sigma}_{c}\left(\theta_{1}\right)\right)
$$

holds for all $\theta_{1}, \theta_{2} \in\left(\theta_{0}-\delta, \theta_{0}+\delta\right)$. Thus, by (4.5),

$$
\left|d\left(\tilde{\mu}_{0}(a), \tilde{\sigma}_{c}\left(\theta_{2}\right)\right)-d\left(\tilde{\mu}_{0}(a), \tilde{\sigma}_{c}\left(\theta_{1}\right)\right)\right| \geq\left(f(c) \sin \varepsilon_{1}\right)\left|\theta_{2}-\theta_{1}\right|
$$

holds for all $a \in\left(a_{0}-\delta, a_{0}+\delta\right), c \in\left(c_{0}-\delta, c_{0}+\delta\right)$, and $\theta_{1}, \theta_{2} \in\left(\theta_{0}-\delta, \theta_{0}+\delta\right)$.

It follows from Lemma 4.1 that, for any positive numbers $a, b$, and $c$ with

$$
|a-c|<b<d\left(\tilde{\mu}_{0}(a), \tilde{\mu}_{\pi}(c)\right),
$$

there exists a geodesic triangle $\triangle(\tilde{p} \tilde{q} \tilde{r})$ in $\widetilde{M}$ such that

$$
d(\tilde{p}, \tilde{q})=a, \quad d(\tilde{q}, \tilde{r})=b, \quad d(\tilde{r}, \tilde{p})=c .
$$

Let $\theta(a, b, c)$ denote the angle $\angle(\tilde{r} \tilde{p} \tilde{q})$ of the triangle $\triangle(\tilde{p} \tilde{q} \tilde{r})$.

Lemma 4.3. The function $\theta(a, b, c)$ defined on

$$
T:=\left\{(a, b, c) \in \mathbb{R}^{3}|a, b, c>0,| a-c \mid<b<d\left(\tilde{\mu}_{0}(a), \tilde{\mu}_{\pi}(c)\right)\right\}
$$

is locally Lipschitz.

Proof. Choose any point $\left(a_{0}, b_{0}, c_{0}\right) \in T$. First, we will prove that

$$
\left|\theta\left(a_{0}+\Delta a, b_{0}, c_{0}\right)-\theta\left(a_{0}, b_{0}, c_{0}\right)\right| \leq \frac{1}{f\left(c_{0}\right) \sin \varepsilon_{1}}|\Delta a|
$$


for all $\Delta a \in \mathbb{R}$ with $|\Delta a|<\delta$. Here, the numbers $\varepsilon_{1}$ and $\delta$ are the constants guaranteed for any $a_{0}, c_{0}$, and $\theta_{0}:=\theta\left(a_{0}, b_{0}, c_{0}\right)$ in Lemma 4.2. It follows from Lemma 4.2 that

$$
\left|d\left(\tilde{\mu}_{0}\left(a_{0}+\Delta a\right), \tilde{\sigma}_{c_{0}}\left(\theta_{0}+\Delta_{a} \theta\right)\right)-d\left(\tilde{\mu}_{0}\left(a_{0}+\Delta a\right), \tilde{\sigma}_{c_{0}}\left(\theta_{0}\right)\right)\right| \geq\left(f\left(c_{0}\right) \sin \varepsilon_{1}\right)\left|\Delta_{a} \theta\right|
$$

for all $\Delta a \in \mathbb{R}$ with $|\Delta a|<\delta$. Here, we set

$$
\Delta_{a} \theta:=\theta\left(a_{0}+\Delta a, b_{0}, c_{0}\right)-\theta_{0}
$$

It is clear that

$$
d\left(\tilde{\mu}_{0}\left(a_{0}+\Delta a\right), \tilde{\sigma}_{c_{0}}\left(\theta_{0}+\Delta_{a} \theta\right)\right)=d\left(\tilde{\mu}_{0}\left(a_{0}\right), \tilde{\sigma}_{c_{0}}\left(\theta_{0}\right)\right)=b_{0} .
$$

It follows from the triangle inequality that

$$
\begin{aligned}
\left|d\left(\tilde{\mu}_{0}\left(a_{0}\right), \tilde{\sigma}_{c_{0}}\left(\theta_{0}\right)\right)-d\left(\tilde{\mu}_{0}\left(a_{0}+\Delta a\right), \tilde{\sigma}_{c_{0}}\left(\theta_{0}\right)\right)\right| & \leq d\left(\tilde{\mu}_{0}\left(a_{0}\right), \tilde{\mu}_{0}\left(a_{0}+\Delta a\right)\right) \\
& =|\Delta a| .
\end{aligned}
$$

By (4.7), (4.8), and (4.9), we get

$$
\left|\Delta_{a} \theta\right| \leq \frac{1}{f\left(c_{0}\right) \sin \varepsilon_{1}}|\Delta a|
$$

for all $\Delta a \in \mathbb{R}$ with $|\Delta a|<\delta$. Thus, the proof of (4.6) is complete. Since

$$
\theta(a, b, c)=\theta(c, b, a)
$$

for all $(a, b, c) \in T$,

$$
\left|\theta\left(a_{0}, b_{0}, c_{0}+\Delta c\right)-\theta\left(a_{0}, b_{0}, c_{0}\right)\right| \leq \frac{1}{f\left(c_{0}\right) \sin \varepsilon_{1}}|\Delta c|
$$

holds for all $\Delta c \in \mathbb{R}$ with $|\Delta c|<\delta$. We omit the proof of the following equation (4.11), since the proof is similar to that of (4.6):

$$
\left|\theta\left(a_{0}, b_{0}+\Delta b, c_{0}\right)-\theta\left(a_{0}, b_{0}, c_{0}\right)\right| \leq \frac{1}{f\left(c_{0}\right) \sin \varepsilon_{1}}|\Delta b|
$$

for all $\Delta b \in \mathbb{R}$ with $|\Delta b|<\delta$. Therefore, the function $\theta(a, b, c)$ is locally Lipschitz at $\left(a_{0}, b_{0}, c_{0}\right) \in T$ by (4.6), (4.10), and (4.11).

Lemma 4.4 (Alexandrov Convexity). Let $(M, p)$ be a complete open Riemannian $n$-manifold $M$ whose radial curvature at the base point $p$ is bounded from below by that of a non-compact model surface of revolution $(\widetilde{M}, \tilde{p})$. For an arbitrary fixed geodesic triangle $\triangle(p x y)$ in $M$, let $x, y:[0,1] \longrightarrow M$ be its edges which are minimal geodesic segments joining $p=x(0)=y(0)$ to $x=x(1), y=y(1)$, respectively, and are parametrized proportionally to arc-length.

Assume that there exists a unique geodesic triangle $\widetilde{\triangle}(p x(t) y(t)):=\triangle(\tilde{p} \tilde{x}(t) \tilde{y}(t))$ in $\widetilde{M}$ up to an isometry corresponding to the triangle $\triangle(p x(t) y(t))$ for each $t \in(0,1)$ such that

$$
d(\tilde{p}, \tilde{x}(t))=d(p, x(t)), d(\tilde{p}, \tilde{y}(t))=d(p, y(t)), d(\tilde{x}(t), \tilde{y}(t))=d(x(t), y(t))
$$

and that

$$
\angle(p x(t) y(t)) \geq \angle(\tilde{p} \tilde{x}(t) \tilde{y}(t)), \quad \angle(p y(t) x(t)) \geq \angle(\tilde{p} \tilde{y}(t) \tilde{x}(t)) .
$$


If $\angle(x p y)<\pi$, then, the function

$$
\theta(t):=\angle(\tilde{x}(t) \tilde{p} \tilde{y}(t))
$$

is locally Lipschitz on $(0,1)$, and non-increasing on $(0,1]$.

Proof. Set

$$
a:=d(p, x), \quad b:=d(x, y), \quad c:=d(p, y) .
$$

Since the edges $x, y:[0,1] \longrightarrow M$ of $\triangle(p x y)$ are parametrized proportionally to arc-length, respectively, we have

$$
a t=d(p, x(t)), \quad c t=d(p, y(t))
$$

for all $t \in[0,1]$. If we define a Lipschitz function on $[0,1]$ as

$$
\varphi(t):=d(x(t), y(t)),
$$

by the assumption (4.12), the function $\theta(t)$ is equal to the function $\theta(a t, \varphi(t), c t)$ by using the function $\theta(\cdot, \cdot, \cdot)$ defined in Lemma 4.3. Hence, $\theta(t)$ is locally Lipschitz by Lemma 4.3. By Dini's theorem [D] (cf. [Hw, Section 2.3], [WZ, Theorem 7.29]), the function $\theta(t)$ is differentiable for almost all $t \in(0,1)$. Thus, we may take any fixed number $t_{0} \in(0,1)$, at which $\theta$ is differentiable. By the assumption (4.13),

$$
\angle\left(p x\left(t_{0}\right) y\left(t_{0}\right)\right) \geq \angle\left(\tilde{p} \tilde{x}\left(t_{0}\right) \tilde{y}\left(t_{0}\right)\right), \quad \angle\left(p y\left(t_{0}\right) x\left(t_{0}\right)\right) \geq \angle\left(\tilde{p} \tilde{y}\left(t_{0}\right) \tilde{x}\left(t_{0}\right)\right) .
$$

Let $\tilde{\mu}, \tilde{\eta}:[0, \infty) \longrightarrow \widetilde{M}$ be meridians emanating from $\tilde{p}$ and passing through $\tilde{x}\left(t_{0}\right)=$ $\tilde{\mu}\left(a t_{0}\right), \tilde{y}\left(t_{0}\right)=\tilde{\eta}\left(c t_{0}\right)$, respectively. Then, we define a function

$$
\widetilde{\psi}(t):=d(\tilde{\mu}(a t), \tilde{\eta}(c t))
$$

on $(0, \infty)$. Observe that

$$
\widetilde{\psi}\left(t_{0}\right)=\varphi\left(t_{0}\right)
$$

holds, since

$$
d\left(\tilde{\mu}\left(a t_{0}\right), \tilde{\eta}\left(c t_{0}\right)\right)=d\left(\tilde{x}\left(t_{0}\right), \tilde{y}\left(t_{0}\right)\right)=d\left(x\left(t_{0}\right), y\left(t_{0}\right)\right) .
$$

Since $\varphi(t)$ and $\widetilde{\psi}(t)$ are Lipschitz functions, respectively, both functions are differentiable almost everywhere. Therefore, we may assume that $\varphi(t)$ and $\widetilde{\psi}(t)$ are also differentiable at $t=t_{0}$. Then,

$$
\varphi^{\prime}\left(t_{0}\right) \leq \widetilde{\psi}^{\prime}\left(t_{0}\right)
$$

holds. To see (4.16), let $q_{0} \in M$ be the midpoint on the edge $x\left(t_{0}\right) y\left(t_{0}\right)$ of $\triangle\left(p x\left(t_{0}\right) y\left(t_{0}\right)\right)$, and let $\sigma, \tau:\left[0, \varphi\left(t_{0}\right) / 2\right] \longrightarrow x\left(t_{0}\right) y\left(t_{0}\right)$ be minimal geodesic segments joining $q_{0}=\sigma(0)=\tau(0)$ to $x\left(t_{0}\right)=\sigma\left(\varphi\left(t_{0}\right) / 2\right), y\left(t_{0}\right)=\tau\left(\varphi\left(t_{0}\right) / 2\right)$, respectively. Then, we see that

$$
\angle\left(\sigma^{\prime}\left(\varphi\left(t_{0}\right) / 2\right), x^{\prime}\left(t_{0}\right)\right)=\angle\left(p x\left(t_{0}\right) y\left(t_{0}\right)\right)
$$

and

$$
\angle\left(\tau^{\prime}\left(\varphi\left(t_{0}\right) / 2\right), y^{\prime}\left(t_{0}\right)\right)=\angle\left(p y\left(t_{0}\right) x\left(t_{0}\right)\right) .
$$

For a sufficiently small fixed number $\varepsilon>0$, consider two geodesic variations of $\sigma$ and $\tau$ whose variational curves join $q_{0}$ to $x(t), y(t)$ for $t \in\left(t_{0}-\varepsilon, t_{0}+\varepsilon\right)$, respectively. 
Since $d\left(q_{0}, x(t)\right)$ and $d\left(q_{0}, y(t)\right)$ are differentiable at $t=t_{0}$, it follows from the triangle inequality, the first variation formula, (4.17), and (4.18) that we have

$$
\begin{aligned}
\varphi^{\prime}\left(t_{0}\right) & =\lim _{t \downarrow t_{0}} \frac{\varphi(t)-\varphi\left(t_{0}\right)}{t-t_{0}} \\
& \leq \lim _{t_{\downarrow} t_{0}} \frac{d\left(q_{0}, x(t)\right)+d\left(q_{0}, y(t)\right)-d\left(q_{0}, x\left(t_{0}\right)\right)-d\left(q_{0}, y\left(t_{0}\right)\right)}{t-t_{0}} \\
& =\lim _{t \downarrow t_{0}} \frac{d\left(q_{0}, x(t)\right)-d\left(q_{0}, x\left(t_{0}\right)\right)}{t-t_{0}}+\lim _{t \downarrow t_{0}} \frac{d\left(q_{0}, y(t)\right)-d\left(q_{0}, y\left(t_{0}\right)\right)}{t-t_{0}} \\
& =\cos \left(\angle\left(p x\left(t_{0}\right) y\left(t_{0}\right)\right)\right)+\cos \left(\angle\left(p y\left(t_{0}\right) x\left(t_{0}\right)\right)\right)
\end{aligned}
$$

and

$$
\begin{aligned}
\varphi^{\prime}\left(t_{0}\right) & =\lim _{t \uparrow t_{0}} \frac{\varphi(t)-\varphi\left(t_{0}\right)}{t-t_{0}} \\
& \geq \lim _{t \uparrow t_{0}} \frac{d\left(q_{0}, x(t)\right)+d\left(q_{0}, y(t)\right)-d\left(q_{0}, x\left(t_{0}\right)\right)-d\left(q_{0}, y\left(t_{0}\right)\right)}{t-t_{0}} \\
& =\cos \left(\angle\left(p x\left(t_{0}\right) y\left(t_{0}\right)\right)\right)+\cos \left(\angle\left(p y\left(t_{0}\right) x\left(t_{0}\right)\right)\right) .
\end{aligned}
$$

Hence, by (4.19) and (4.20), we get

$$
\varphi^{\prime}\left(t_{0}\right)=\cos \left(\angle\left(p x\left(t_{0}\right) y\left(t_{0}\right)\right)\right)+\cos \left(\angle\left(p y\left(t_{0}\right) x\left(t_{0}\right)\right)\right) .
$$

By the same way as above, we see that

$$
\widetilde{\psi}^{\prime}\left(t_{0}\right)=\cos \left(\angle\left(\tilde{p} \tilde{x}\left(t_{0}\right) \tilde{y}\left(t_{0}\right)\right)\right)+\cos \left(\angle\left(\tilde{p} \tilde{y}\left(t_{0}\right) \tilde{x}\left(t_{0}\right)\right)\right) .
$$

Thus, by (4.14), (4.21), and (4.22), we get (4.16), that is,

$$
\begin{aligned}
\varphi^{\prime}\left(t_{0}\right) & =\cos \left(\angle\left(p x\left(t_{0}\right) y\left(t_{0}\right)\right)\right)+\cos \left(\angle\left(p y\left(t_{0}\right) x\left(t_{0}\right)\right)\right) \\
& \leq \cos \left(\angle\left(\tilde{p} \tilde{x}\left(t_{0}\right) \tilde{y}\left(t_{0}\right)\right)\right)+\cos \left(\angle\left(\tilde{p} \tilde{y}\left(t_{0}\right) \tilde{x}\left(t_{0}\right)\right)\right)=\widetilde{\psi}^{\prime}\left(t_{0}\right) .
\end{aligned}
$$

Therefore, we get

$$
\theta^{\prime}\left(t_{0}\right) \leq 0
$$

by (4.16). Indeed, suppose that

$$
\theta^{\prime}\left(t_{0}\right)>0
$$

Then, there exists a constant $\delta>0$ such that

$$
\theta(t)-\theta\left(t_{0}\right) \geq \delta\left(t-t_{0}\right)
$$

holds for all $t>t_{0}$ sufficiently close to $t_{0}$. By considering the point $\tilde{\mu}(a t)$ as the vertex $\tilde{x}(t)$ of the geodesic triangle $\triangle(\tilde{p} \tilde{x}(t) \tilde{y}(t))$ corresponding to the geodesic triangle $\triangle(p x(t) y(t))$, it follows from Lemma 4.2 and (4.23) that there exists a constant $\varepsilon_{1} \in(0, \pi / 2)$ such that

$$
\begin{aligned}
\varphi(t)=d(x(t), y(t)) & =d(\tilde{\mu}(a t), \tilde{y}(t)) \\
& \geq d(\tilde{\mu}(a t), \tilde{\eta}(c t))+\left(f(c t) \sin \varepsilon_{1}\right)\left(\theta(t)-\theta\left(t_{0}\right)\right) \\
& \geq \widetilde{\psi}(t)+\left(\delta f(c t) \sin \varepsilon_{1}\right)\left(t-t_{0}\right)
\end{aligned}
$$


for all $t>t_{0}$ sufficiently close to $t_{0}$. By (4.15) and (4.24),

$$
\varphi(t)-\varphi\left(t_{0}\right) \geq \widetilde{\psi}(t)-\widetilde{\psi}\left(t_{0}\right)+\left(\delta f(c t) \sin \varepsilon_{1}\right)\left(t-t_{0}\right) .
$$

Hence, by (4.25), we get

$$
\varphi^{\prime}\left(t_{0}\right) \geq \widetilde{\psi}^{\prime}\left(t_{0}\right)+\delta f(c t) \sin \varepsilon_{1}>\widetilde{\psi}^{\prime}\left(t_{0}\right) .
$$

This is a contradiction, since $\varphi^{\prime}\left(t_{0}\right) \leq \widetilde{\psi^{\prime}}\left(t_{0}\right)$. Thus, $\theta^{\prime}(t) \leq 0$ for almost all $t \in$ $(0,1)$. This implies that $\theta(t)$ is non-increasing on $(0,1]$.

Remark 4.5. If the function $\theta(t)$ in Lemma 4.4 is not locally Lipschitz, then we can not conclude that $\theta$ is non-increasing. For example, the Cantor-Lebesgue function is increasing on $[0,1]$ and its derivative function is zero almost everywhere. By making use of this function, we may construct a function which is not increasing, or decreasing, but its derivative function is zero almost everywhere (cf. WZ]).

4.2. Toponogov comparison theorem. We are going to show our Toponogov comparison theorem (Theorem 4.12). We first introduce the definition of a narrow geodesic triangle in an arbitrary complete Riemannian manifold with a base point.

Definition 4.6 ([IMS, Section 2]). Let $M$ be a complete Riemannian manifold with a base point $p \in M$. A geodesic triangle $\triangle(p x y)$ in $M$ is called a narrow geodesic triangle if

$$
d(x, y) \ll \min \{d(p, x), d(p, y)\}
$$

and the Fermi coordinates around the edge $p x$ (respectively $p y$ ) contains the edge $p y$ (respectively $p x$ ).

From the Rauch-Berger comparison theorem, we have the following lemma on a narrow geodesic triangle.

Lemma 4.7 ([IMS, Lemma 2.2]). Let $(M, p)$ be a complete open Riemannian nmanifold $M$ whose radial curvature at the base point $p$ is bounded from below by that of a non-compact model surface of revolution $(\widetilde{M}, \tilde{p})$. Then, for every narrow geodesic triangle $\triangle(p x y)$ in $M$, there exists a geodesic triangle $\widetilde{\triangle}(p x y):=\triangle(\tilde{p} \tilde{x} \tilde{y})$ in $\widetilde{M}$ such that

$$
d(\tilde{p}, \tilde{x})=d(p, x), \quad d(\tilde{p}, \tilde{y})=d(p, y), \quad d(\tilde{x}, \tilde{y})=d(x, y)
$$

and that

$$
\angle(p x y) \geq \angle(\tilde{p} \tilde{x} \tilde{y}), \quad \angle(p y x) \geq \angle(\tilde{p} \tilde{y} \tilde{x}) .
$$

Remark 4.8. Another proof of Lemma 4.7 is found in [KT2]. Here, from the standpoint of radial curvature geometry, we establish the Toponogov comparison theorem for open triangles on complete manifolds with boundary. In particular, it will be clarified in [KT2] that the cut locus of a complete open Riemannian manifold $M$ is not an obstruction at all when we draw a corresponding geodesic triangle in a model surface of revolution for each geodesic triangle in the manifold $M$.

Then, we prove the next lemma by using Lemmas 4.1 and 4.7 .

Lemma 4.9. Let $(M, p)$ be a complete open Riemannian n-manifold $M$ whose radial curvature at the base point $p$ is bounded from below by that of a non-compact 
model surface of revolution $(\widetilde{M}, \tilde{p})$. If the geodesic triangle $\triangle(p x y)$ in $M$ admits a geodesic triangle $\widetilde{\triangle}(p x y):=\triangle(\tilde{p} \tilde{x} \tilde{y})$ in $\widetilde{V}\left(\delta_{0}\right)$ for some $\delta_{0} \in(0, \pi)$ satisfying

$$
d(\tilde{p}, \tilde{x})=d(p, x), \quad d(\tilde{p}, \tilde{y})=d(p, y), \quad d(\tilde{x}, \tilde{y})=d(x, y),
$$

then, for any $s \in(0, \ell)$, the geodesic triangle $\triangle(p x \sigma(s))$ has a geodesic triangle $\widetilde{\triangle}(p x \sigma(s))$ in $\widetilde{V}\left(\delta_{0}\right)$ satisfying (4.28) for $y=\sigma(s)$, where $\sigma:[0, \ell] \longrightarrow M$ is the minimal geodesic segment joining $\sigma(0)=x$ to $\sigma(\ell)=y$.

Proof. We consider the set $S$ consisting of all $s \in(0, \ell)$ such that, for any $r \in(0, s)$, there exists a geodesic triangle $\widetilde{\triangle}(p x \sigma(r)):=\triangle(\tilde{p} \tilde{x} \tilde{\sigma}(r)) \subset \widetilde{V}\left(\delta_{0}\right)$ corresponding to the triangle $\triangle(p x \sigma(r)) \subset M$ satisfying (4.28) for $y=\sigma(r)$. It is clear from Lemma 4.7 that $S$ is a non-empty set. By supposing

$$
s_{1}:=\sup S<\ell,
$$

we will get a contradiction. By definition, there exists a geodesic triangle $\widetilde{\triangle}\left(p x \sigma\left(s_{1}\right)\right)$ $\subset \widetilde{M}$ such that

$$
\angle\left(\tilde{x} \tilde{p} \tilde{\sigma}\left(s_{1}\right)\right) \geq \delta_{0}
$$

and (4.28) is valid for $y=\sigma\left(s_{1}\right)$. From Lemma 4.7, we may take a subdivision

$$
0=s_{0}<s_{1}<\cdots<s_{k}=\ell
$$

of $[0, \ell]$ containing the $s_{1}$ such that all the triangles

$$
\triangle\left(p z_{i} z_{i+1}\right):=\triangle\left(p \sigma\left(s_{i}\right) \sigma\left(s_{i+1}\right)\right) \subset M
$$

have corresponding triangles $\widetilde{\triangle}\left(p z_{i} z_{i+1}\right) \subset \widetilde{M}$ satisfying (4.28) for $x=z_{i}$ and $y=z_{i+1}$, respectively. Here, we set $z_{i}:=\sigma\left(s_{i}\right)$. Under this situation, for $i=1$, we draw $\widetilde{\triangle}\left(p z_{1} z_{2}\right)$ on $\widetilde{M}$, which is adjacent to $\widetilde{\triangle}\left(p z_{0} z_{1}\right)$, so as to have a common edge $\tilde{p} \tilde{z}_{1}$. Inductively, we draw $\widetilde{\triangle}\left(p z_{i} z_{i+1}\right)$ on $\widetilde{M}$, which is adjacent to $\widetilde{\triangle}\left(p z_{i-1} z_{i}\right)$, so as to have a common edge $\tilde{p} \tilde{z}_{i}$. Hence, we get a broken geodesic $\tilde{\eta}$ emanating from $\tilde{x}=\tilde{z}_{0}$ to $\tilde{z}_{k}$, which consists of the opposite sides $\tilde{z}_{i} \tilde{z}_{i+1}$ to $\tilde{p}$. It is trivial that the length $L(\tilde{\eta})$ of $\tilde{\eta}$ is equal to

$$
L(\tilde{\eta})=\sum_{i=1}^{k} d\left(\tilde{z}_{i-1}, \tilde{z}_{i}\right)=d(x, y) .
$$

Suppose that the sum of the angles is

$$
\sum_{i=1}^{k} \angle\left(\tilde{z}_{i-1} \tilde{p} \tilde{z}_{i}\right) \leq \pi
$$

Since

$$
\sum_{i=1}^{k} \angle\left(\tilde{z}_{i-1} \tilde{p} \tilde{z}_{i}\right)>\angle\left(\tilde{x} \tilde{p} \tilde{\sigma}\left(s_{1}\right)\right) \geq \delta_{0}>\angle(\tilde{x} \tilde{y} \tilde{y})
$$

by (4.29), it follows from Lemma 4.1 and (4.30) that

$$
d(\tilde{x}, \tilde{y})<d\left(\tilde{x}, \tilde{z}_{k}\right) \leq L(\tilde{\eta})=d(x, y) .
$$

This is a contradiction, since $d(\tilde{x}, \tilde{y})=d(x, y)$. Hence, we see that the sum of the angles is

$$
\sum_{i=1}^{k} \angle\left(\tilde{z}_{i-1} \tilde{p} \tilde{z}_{i}\right)>\pi \text {. }
$$


Without loss of generality, we may assume that $\theta(\tilde{x})=0$ and $\theta \circ \tilde{\eta}$ is increasing. Since $\tilde{\eta}$ intersects the meridian $\tilde{\mu}_{\pi}$ defined by $\theta=\pi$, we get a unique intersection $\tilde{w}_{0}$. The point $\tilde{w}_{0}$ divides $\tilde{\eta}$ into two broken geodesics $\tilde{\eta}_{1}$ and $\tilde{\eta}_{2}$. Hence, we assume that the subarc emanating from $\tilde{x}=\tilde{z}_{0}$ to $\tilde{w}_{0}$ is $\tilde{\eta}_{1}$. Since the point $\tilde{\mu}_{\pi}(d(\tilde{p}, \tilde{y}))$ is the nearest point to $\tilde{w}_{0}$ on the parallel $t=d(\tilde{p}, \tilde{y})$, we have

$$
L(\tilde{\eta})=L\left(\tilde{\eta}_{1}\right)+L\left(\tilde{\eta}_{2}\right)>L\left(\tilde{\eta}_{1}\right)+d\left(\tilde{w}_{0}, \tilde{\mu}_{\pi}(d(\tilde{p}, \tilde{y}))\right) .
$$

Thus, the broken geodesic $\tilde{\xi}$ consisting of $\tilde{\eta}_{1}$ and the minimal geodesic segment joining $\tilde{w}_{0}$ to $\tilde{\mu}_{\pi}(d(\tilde{p}, \tilde{y}))$ is shorter than $\tilde{\eta}$. Since

$$
L(\tilde{\xi}) \geq d\left(\tilde{x}, \tilde{\mu}_{\pi}(d(\tilde{p}, \tilde{y}))\right)
$$

we have, by (4.31),

$$
L(\tilde{\eta})>d\left(\tilde{x}, \tilde{\mu}_{\pi}(d(\tilde{p}, \tilde{y}))\right)
$$

From Lemma 4.1

$$
d\left(\tilde{x}, \tilde{\mu}_{\pi}(d(\tilde{p}, \tilde{y}))\right)>d(\tilde{x}, \tilde{y})
$$

holds, since $\pi>\delta_{0}>\angle(\tilde{x} \tilde{p} \tilde{y})$. Hence, by (4.32) and (4.33), we have

$$
L(\tilde{\eta})>d(\tilde{x}, \tilde{y}) .
$$

Since $L(\tilde{\eta})=d(x, y)=d(\tilde{x}, \tilde{y})$, we get a contradiction from (4.34). Therefore, the proof is complete.

Lemma 4.10. Assume that a non-compact model surface of revolution $(\widetilde{M}, \tilde{p})$ with a metric (1.1) admits a sector $\widetilde{V}\left(\delta_{0}\right)$ for some $\delta_{0} \in(0, \pi)$ which has no pair of cut points. Let $\triangle\left(\tilde{p} \tilde{x}_{1} \tilde{y}_{1}\right), \triangle\left(\tilde{p} \tilde{x}_{2} \tilde{y}_{2}\right)$ be geodesic triangles in $\widetilde{M}$ satisfying

$$
d\left(\tilde{p}, \tilde{y}_{1}\right)=d\left(\tilde{p}, \tilde{x}_{2}\right)
$$

and

$$
\angle\left(\tilde{p} \tilde{y}_{1} \tilde{x}_{1}\right)+\angle\left(\tilde{p} \tilde{x}_{2} \tilde{y}_{2}\right) \leq \pi .
$$

If there exists a geodesic triangle $\triangle(\tilde{p} \tilde{x} \tilde{y})$ in $\widetilde{V}\left(\delta_{0}\right)$ such that

$$
d(\tilde{p}, \tilde{x})=d\left(\tilde{p}, \tilde{x}_{1}\right), \quad d(\tilde{p}, \tilde{y})=d\left(\tilde{p}, \tilde{y}_{2}\right),
$$

and that

$$
d(\tilde{x}, \tilde{y})=d\left(\tilde{x}_{1}, \tilde{y}_{1}\right)+d\left(\tilde{x}_{2}, \tilde{y}_{2}\right)
$$

then,

$$
\angle\left(\tilde{p} \tilde{x}_{1} \tilde{y}_{1}\right) \geq \angle(\tilde{p} \tilde{x} \tilde{y}), \quad \angle\left(\tilde{p} \tilde{y}_{2} \tilde{x}_{2}\right) \geq \angle(\tilde{p} \tilde{y} \tilde{x}) .
$$

Proof. From (4.35), we may draw $\triangle\left(\tilde{p} \tilde{x}_{2} \tilde{y}_{2}\right)$ on $\widetilde{M}$, which is adjacent to $\triangle\left(\tilde{p} \tilde{x}_{1} \tilde{y}_{1}\right)$, so as to have a common edge $\tilde{p} \tilde{y}_{1}=\tilde{p} \tilde{x}_{2}$, i.e., $\tilde{y}_{1}=\tilde{x}_{2}$. For the metric (1.1), we set

$$
0:=\theta\left(\tilde{x}_{1}\right)<\theta\left(\tilde{y}_{1}\right)=\theta\left(\tilde{x}_{2}\right)<\theta\left(\tilde{y}_{2}\right) .
$$

Since the existence of $\triangle(\tilde{p} \tilde{x} \tilde{y}) \subset \widetilde{V}\left(\delta_{0}\right)$ is unique up to an isometry fixing $\tilde{p}$, we may assume, by $d(\tilde{p}, \tilde{x})=d\left(\tilde{p}, \tilde{x}_{1}\right)$ of (4.37), that

$$
0=\theta(\tilde{x})<\theta(\tilde{y})
$$

i.e., $\tilde{x}=\tilde{x}_{1}$ and $\theta(\tilde{y})>0$. Since $\theta(\tilde{y})<\delta_{0}<\pi$, it is clear from the same argument in the proof of Lemma 4.9 that

$$
\theta\left(\tilde{y}_{2}\right) \leq \pi
$$


holds. By the triangle inequality and (4.38),

$$
d\left(\tilde{x}_{1}, \tilde{y}_{2}\right) \leq d\left(\tilde{x}_{1}, \tilde{y}_{1}\right)+d\left(\tilde{y}_{1}, \tilde{y}_{2}\right)=d(\tilde{x}, \tilde{y})=d\left(\tilde{x}_{1}, \tilde{y}\right) .
$$

Since $\triangle(\tilde{p} \tilde{x} \tilde{y}) \subset \widetilde{V}\left(\delta_{0}\right)$, (4.40), and (4.41), it follows from Lemma 4.1 that $\theta\left(\tilde{y}_{2}\right) \leq$ $\angle\left(\tilde{x}_{1} \tilde{p} \tilde{y}\right)<\delta_{0}$ holds, i.e.,

$$
\tilde{y}_{2} \in \theta^{-1}\left(0, \delta_{0}\right) .
$$

Let $\widetilde{\mathcal{D}}\left(\delta_{0}\right)$ denote the domain defined by

$$
\widetilde{\mathcal{D}}\left(\delta_{0}\right):=\left\{\tilde{q} \in \theta^{-1}\left(0, \delta_{0}\right) \mid t(\tilde{q})<t(\tilde{\sigma}(s)) \text { whenever } \theta(\tilde{q})=\theta(\tilde{\sigma}(s)) \text { for some } s>0\right\} .
$$

Here $\tilde{\sigma}:[0, \infty) \longrightarrow \widetilde{M}$ denotes the unit speed geodesic emanating from $\tilde{x}_{1}$ passing through $\tilde{y}_{1}=\tilde{\sigma}\left(d\left(\tilde{x}_{1}, \tilde{y}_{1}\right)\right)$. Thus, the domain $\widetilde{\mathcal{D}}\left(\delta_{0}\right)$ is bounded by the three subarcs of the two meridians $\theta=0, \theta=\delta_{0}$, and $\tilde{\sigma}$.

There is nothing to prove, if $\angle\left(\tilde{p} \tilde{y}_{1} \tilde{x}_{1}\right)+\angle\left(\tilde{p} \tilde{y}_{1} \tilde{y}_{2}\right)=\pi$ holds. Thus, from (4.36), we may assume that

$$
\angle\left(\tilde{p} \tilde{y}_{1} \tilde{x}_{1}\right)+\angle\left(\tilde{p} \tilde{y}_{1} \tilde{y}_{2}\right)<\pi .
$$

Hence, by (4.42) and (4.43), it is clear that $\tilde{y}_{2} \in \widetilde{\mathcal{D}}\left(\delta_{0}\right)$. Let $\tilde{c}:\left[\theta\left(\tilde{y}_{2}\right), \delta_{0}\right] \longrightarrow \widetilde{M}$ denote the subarc of the parallel $t=d\left(\tilde{p}, \tilde{y}_{2}\right)$ that is cut off by two meridians $\theta=$ $\theta\left(\tilde{y}_{2}\right)$ and $\theta=\delta_{0}$. Here $\tilde{c}$ is assumed to be parametrized by $\theta$, so that $\left.\tilde{c}\right|_{\left[\theta\left(\tilde{y}_{2}\right), \delta_{0}\right)} \subset$ $\widetilde{V}\left(\delta_{0}\right)$. From Lemma 4.1, $d(\tilde{p}, \tilde{y})=d\left(\tilde{p}, \tilde{y}_{2}\right)$ of (4.37), and (4.41), it follows that

$$
d\left(\tilde{x}_{1}, \tilde{c}\left(\theta\left(\tilde{y}_{2}\right)\right)\right)=d\left(\tilde{x}_{1}, \tilde{y}_{2}\right) \leq d\left(\tilde{x}_{1}, \tilde{c}(\theta)\right) \leq d\left(\tilde{x}_{1}, \tilde{c}\left(\delta_{0}\right)\right)
$$

and

$$
d\left(\tilde{x}_{1}, \tilde{y}_{2}\right)<d\left(\tilde{x}_{1}, \tilde{y}\right) .
$$

Moreover, it follows from (4.38), (4.45), and $\triangle(\tilde{p} \tilde{x} \tilde{y}) \subset \tilde{V}\left(\delta_{0}\right)$ that

$$
d\left(\tilde{x}_{1}, \tilde{c}\left(\delta_{0}\right)\right)>d\left(\tilde{x}_{1}, \tilde{y}\right)=d\left(\tilde{x}_{1}, \tilde{y}_{1}\right)+d\left(\tilde{y}_{1}, \tilde{y}_{2}\right)>d\left(\tilde{x}_{1}, \tilde{y}_{2}\right) .
$$

Therefore, by (4.44), (4.46), and the intermediate value theorem, there exists a point $\tilde{y}_{3} \in \theta^{-1}\left(\theta\left(\tilde{y}_{2}\right), \delta_{0}\right)$ on $\left.\tilde{c}\right|_{\left(\theta\left(\tilde{y}_{2}\right), \delta_{0}\right)}$ such that

$$
d\left(\tilde{x}_{1}, \tilde{y}_{3}\right)=d\left(\tilde{x}_{1}, \tilde{y}_{1}\right)+d\left(\tilde{y}_{1}, \tilde{y}_{2}\right) .
$$

Supposing that $\tilde{y}_{3}$ is not a point in the closure of $\widetilde{\mathcal{D}}\left(\delta_{0}\right)$, we will get a contradiction. Since $\tilde{y}_{2} \in \widetilde{\mathcal{D}}\left(\delta_{0}\right)$ and $\tilde{y}_{3}$ is not in the closure of $\widetilde{\mathcal{D}}\left(\delta_{0}\right), \tilde{\sigma}$ intersects $\tilde{c}$ at a point $\tilde{c}\left(\theta_{0}\right)=\tilde{\sigma}\left(s_{0}\right)$. Without loss of generality, we may assume that $\left.\tilde{c}\right|_{\left[\theta\left(\tilde{y}_{2}\right), \theta_{0}\right)}$ lies in $\widetilde{\mathcal{D}}\left(\delta_{0}\right)$. From Lemma 4.1, it follows that

$$
d\left(\tilde{y}_{1}, \tilde{y}_{2}\right)<d\left(\tilde{y}_{1}, \tilde{c}\left(\theta_{0}\right)\right)=d\left(\tilde{y}_{1}, \tilde{\sigma}\left(s_{0}\right)\right) .
$$

Since $\left.\tilde{\sigma}\right|_{\left[0, s_{0}\right]}$ is minimal, it follows from (4.48) that

$$
d\left(\tilde{x}_{1}, \tilde{c}\left(\theta_{0}\right)\right)=d\left(\tilde{x}_{1}, \tilde{y}_{1}\right)+d\left(\tilde{y}_{1}, \tilde{c}\left(\theta_{0}\right)\right)>d\left(\tilde{x}_{1}, \tilde{y}_{1}\right)+d\left(\tilde{y}_{1}, \tilde{y}_{2}\right) .
$$

By using Lemma 4.1 again,

$$
d\left(\tilde{x}_{1}, \tilde{c}\left(\theta_{0}\right)\right)<d\left(\tilde{x}_{1}, \tilde{y}_{3}\right) .
$$

Combining (4.49) and (4.50), we get

$$
d\left(\tilde{x}_{1}, \tilde{y}_{3}\right)>d\left(\tilde{x}_{1}, \tilde{y}_{1}\right)+d\left(\tilde{y}_{1}, \tilde{y}_{2}\right) .
$$

This is a contradiction to (4.47). Thus, $\tilde{y}_{3}$ lies in the closure of $\widetilde{\mathcal{D}}\left(\delta_{0}\right)$. Since any subarc of $\tilde{\sigma}$ is minimal when the subarc lies in $\theta^{-1}\left(0, \delta_{0}\right)$, the minimal geodesic 
joining $\tilde{x}_{1}$ to $\tilde{y}_{3}$ lies in the closure of $\widetilde{\mathcal{D}}\left(\delta_{0}\right)$. Then, we get a geodesic triangle $\triangle\left(\tilde{p} \tilde{x}_{1} \tilde{y}_{3}\right) \subset \tilde{V}\left(\delta_{0}\right)$ satisfying (4.37) and (4.38) for $\tilde{x}=\tilde{x}_{1}$ and $\tilde{y}=\tilde{y}_{3}$. Since $\widetilde{V}\left(\delta_{0}\right)$ has no pair of cut points, $\triangle(\tilde{p} \tilde{x} \tilde{y})$ and $\triangle\left(\tilde{p} \tilde{x}_{1} \tilde{y}_{3}\right)$ are isometric, i.e., $\triangle(\tilde{p} \tilde{x} \tilde{y})=$ $\triangle\left(\tilde{p} \tilde{x}_{1} \tilde{y}_{3}\right)$. Therefore, $\triangle(\tilde{p} \tilde{x} \tilde{y})$ satisfies

$$
\angle\left(\tilde{p} \tilde{x}_{1} \tilde{y}_{1}\right) \geq \angle(\tilde{p} \tilde{x} \tilde{y})
$$

of (4.39), because the minimal geodesic joining $\tilde{x}=\tilde{x}_{1}$ to $\tilde{y}=\tilde{y}_{3}$ lies in the closure of $\widetilde{\mathcal{D}}\left(\delta_{0}\right)$. By exchanging $\tilde{x}$ and $\tilde{y}$, and doing $\tilde{x}_{1}$ and $\tilde{y}_{2}$, respectively, in the argument above, we may get a geodesic triangle $\triangle\left(\tilde{p} \tilde{x}_{3} \tilde{y}_{2}\right) \subset \widetilde{V}\left(\delta_{0}\right)$ satisfying (4.37) and (4.38) for $\tilde{x}=\tilde{x}_{3}$ and $\tilde{y}=\tilde{y}_{2}$, and $\angle\left(\tilde{p} \tilde{y}_{2} \tilde{x}_{2}\right) \geq \angle\left(\tilde{p} \tilde{y}_{2} \tilde{x}_{3}\right)$. Since both triangles $\triangle(\tilde{p} \tilde{x} \tilde{y}), \triangle\left(\tilde{p} \tilde{x}_{3} \tilde{y}_{2}\right) \subset \tilde{V}\left(\delta_{0}\right)$ are isometric, $\angle(\tilde{p} \tilde{y} \tilde{x})=\angle\left(\tilde{p} \tilde{y}_{2} \tilde{x}_{3}\right)$ holds; i.e., we get

$$
\angle\left(\tilde{p} \tilde{y}_{2} \tilde{x}_{2}\right) \geq \angle(\tilde{p} \tilde{y} \tilde{x})
$$

of (4.39). Therefore, we have proved our lemma.

By Lemmas 4.7, 4.9, and 4.10, we have the next lemma.

Lemma 4.11 (Essential Lemma for Toponogov comparison theorem). Let ( $M, p)$ be a complete open Riemannian n-manifold $M$ whose radial curvature at the base point $p$ is bounded from below by that of a non-compact model surface of revolution $(\widetilde{M}, \tilde{p})$. Assume that $\widetilde{M}$ admits a sector $\widetilde{V}\left(\delta_{0}\right)$ for some $\delta_{0} \in(0, \pi)$ which has no pair of cut points. If a geodesic triangle $\triangle(p x y)$ in $M$ admits a geodesic triangle $\widetilde{\triangle}(p x y):=\triangle(\tilde{p} \tilde{x} \tilde{y})$ in $\widetilde{V}\left(\delta_{0}\right)$ satisfying

$$
d(\tilde{p}, \tilde{x})=d(p, x), \quad d(\tilde{p}, \tilde{y})=d(p, y), \quad d(\tilde{x}, \tilde{y})=d(x, y),
$$

then

$$
\angle(p x y) \geq \angle(\tilde{p} \tilde{x} \tilde{y}), \quad \angle(p y x) \geq \angle(\tilde{p} \tilde{y} \tilde{x}) .
$$

Proof. Let $\triangle(p x y)$ denote any geodesic triangle in $M$ which admits a geodesic triangle $\widetilde{\triangle}(p x y)$ in $\widetilde{V}\left(\delta_{0}\right)$ satisfying (4.51). We denote by $\sigma:[0, \ell] \longrightarrow M$ the edge of $\triangle(p x y)$ opposite to $p$. Let $S$ be the set of all $r \in(0, \ell]$ such that there exists a geodesic triangle $\widetilde{\triangle}(p x \sigma(r)):=\triangle(\tilde{p} \tilde{x} \tilde{\sigma}(r)) \subset \widetilde{V}\left(\delta_{0}\right)$ corresponding to the triangle $\triangle(p x \sigma(r)) \subset M$ satisfying (4.51) and (4.52) for $y=\sigma(r)$. It is clear from Lemma 4.7 that $S$ is non-empty. Since there is nothing to prove in the case where $\sup S=\ell$, we suppose that

$$
s_{1}:=\sup S<\ell .
$$

By Lemma 4.9, there exists a geodesic triangle $\widetilde{\triangle}\left(p x \sigma\left(s_{1}\right)\right) \subset \widetilde{V}\left(\delta_{0}\right)$ corresponding to the triangle $\triangle\left(p x \sigma\left(s_{1}\right)\right) \subset M$ satisfying (4.51) and (4.52) for $y=\sigma\left(s_{1}\right)$. For a sufficiently small $\ell-s_{1}>\varepsilon>0$, it follows from Lemma 4.7 that there exists a geodesic triangle $\widetilde{\triangle}\left(p \sigma\left(s_{1}\right) \sigma\left(s_{1}+\varepsilon\right)\right)$ corresponding to the triangle $\triangle\left(p \sigma\left(s_{1}\right) \sigma\left(s_{1}+\varepsilon\right)\right) \subset M$ satisfying (4.51) and (4.52) for $x=\sigma\left(s_{1}\right)$ and $y=\sigma\left(s_{1}+\varepsilon\right)$. Thus, two geodesic triangles $\widetilde{\triangle}\left(p x \sigma\left(s_{1}\right)\right)$ and $\widetilde{\triangle}\left(p \sigma\left(s_{1}\right) \sigma\left(s_{1}+\varepsilon\right)\right)$ satisfy (4.35) and (4.36) (in Lemma 4.10) for $\tilde{x}_{1}=\tilde{x}, \tilde{y}_{1}=\tilde{\sigma}\left(s_{1}\right), \tilde{x}_{2}=\tilde{\sigma}\left(s_{1}\right)$, and $\tilde{y}_{2}=\tilde{\sigma}\left(s_{1}+\varepsilon\right)$. On the other hand, by Lemma 4.9 again, we get a geodesic triangle $\triangle(\tilde{x} \tilde{p} \hat{y}) \subset \widetilde{V}\left(\delta_{0}\right)$ satisfying (4.37) and (4.38) (in Lemma 4.10) for $\tilde{y}=\hat{y}, \tilde{x}_{1}=\tilde{x}, \tilde{y}_{1}=\tilde{\sigma}\left(s_{1}\right)$, $\tilde{x}_{2}=\tilde{\sigma}\left(s_{1}\right)$, and $\tilde{y}_{2}=\tilde{\sigma}\left(s_{1}+\varepsilon\right)$. Thus, it follows from Lemma 4.10 that $s_{1}+\varepsilon \in S$. This contradicts the fact that $s_{1}$ is the supremum of $S$.

Then, by Lemmas 4.4 and 4.11 we have a new type of Toponogov comparison theorem, which is the main theorem in this section. 
Theorem 4.12 (A new type of Toponogov comparison theorem). Let $(M, p)$ be a complete open Riemannian n-manifold $M$ whose radial curvature at the base point $p$ is bounded from below by that of a non-compact model surface of revolution $(\widetilde{M}, \tilde{p})$. Assume that $\widetilde{M}$ admits a sector $\widetilde{V}\left(\delta_{0}\right)$ for some $\delta_{0} \in(0, \pi)$ which has no pair of cut points. Then, for every geodesic triangle $\triangle(p x y)$ in $M$ with $\angle(x p y)<\delta_{0}$, there exists a geodesic triangle $\widetilde{\triangle}(p x y):=\triangle(\tilde{p} \tilde{x} \tilde{y})$ in $\widetilde{V}\left(\delta_{0}\right)$ such that

$$
d(\tilde{p}, \tilde{x})=d(p, x), \quad d(\tilde{p}, \tilde{y})=d(p, y), \quad d(\tilde{x}, \tilde{y})=d(x, y)
$$

and that

$$
\angle(x p y) \geq \angle(\tilde{x} \tilde{p} \tilde{y}), \quad \angle(p x y) \geq \angle(\tilde{p} \tilde{x} \tilde{y}), \quad \angle(p y x) \geq \angle(\tilde{p} \tilde{y} \tilde{x}) .
$$

Proof. Let $W$ be the set of all $t \in(0,1)$ such that there exists a geodesic triangle $\widetilde{\triangle}(p x(t) y(t)):=\triangle(\tilde{p} \tilde{x}(t) \tilde{y}(t)) \subset \widetilde{V}\left(\delta_{0}\right)$ corresponding to the triangle $\triangle(p x(t) y(t)) \subset$ $M$ satisfying (4.53) for $x=x(t)$ and $y=y(t)$. Here $x(t)$ and $y(t)$ denote the minimal geodesic segments introduced in Lemma 4.4, respectively. It is clear that $W$ is open. From the Rauch comparison theorem, there exists a constant $\varepsilon_{0}>0$ such that, for each $t \in\left(0, \varepsilon_{0}\right)$, there exists a geodesic triangle $\widetilde{\triangle}(p x(t) y(t)) \subset \widetilde{V}\left(\delta_{0}\right)$ corresponding to $\triangle(p x(t) y(t)) \subset M$ satisfying (4.53) for $x=x(t)$ and $y=y(t)$ and

$$
\theta(t):=\angle(\tilde{x}(t) \tilde{p} \tilde{y}(t)) \leq \angle(x(t) p y(t))=\angle(x p y)<\delta_{0} .
$$

Hence we get

$$
\left(0, \varepsilon_{0}\right) \subset W .
$$

Let $\left(0, t_{0}\right) \subset W$ denote the connected component of $W$ containing $\left(0, \varepsilon_{0}\right)$. From Lemmas 4.4 and 4.11, it follows that $\theta(t)$ is non-increasing on $\left(0, t_{0}\right)$. Thus, the geodesic triangle $\triangle\left(p x\left(t_{0}\right) y\left(t_{0}\right)\right)$ has a corresponding triangle $\widetilde{\triangle}\left(p x\left(t_{0}\right) y\left(t_{0}\right)\right) \subset$ $\widetilde{V}\left(\delta_{0}\right)$ satisfying (4.53) for $x=x\left(t_{0}\right)$ and $y=y\left(t_{0}\right)$. This implies that $t_{0} \in W$ if $t_{0}<1$. Therefore, $W=(0,1)$ and the proof is complete.

Remark 4.13. We refer to [03 for a generalization of the Toponogov comparison theorems to the Finsler geometry and its applications.

\section{Application of the Main Theorem}

In this section, we first give the proof of the Model Lemma (Lemma 5.1). After recalling some differential inequality (Lemma 5.2), we give the proof of our answer (Theorem 5.3) to Milnor's open conjecture. Finally, we prove a corollary (Corollary 5.4) to the Main Theorem.

5.1. Proof of the Model Lemma. Let $M$ be an arbitrary complete open Riemannian $n$-manifold. Fix any point $p \in M$. Then, we have

Lemma 5.1. There exists a locally Lipschitz function $G(t)$ on $[0, \infty)$ such that the radial curvature of $M$ at $p$ is bounded from below by that of the non-compact model surface of revolution with radial curvature function $G(t)$.

Proof. We set

$$
\mathbb{S}_{p}^{n-1}:=\left\{v \in T_{p} M \mid\|v\|=1\right\} .
$$

Let $\gamma_{v}:[0, \rho(v)] \longrightarrow M$ be a minimal geodesic emanating from $p=\gamma_{v}(0)$ such that $v=\gamma_{v}^{\prime}(0) \in \mathbb{S}_{p}^{n-1}$, where we set

$$
\rho(v):=\sup \left\{t>0 \mid d\left(p, \gamma_{v}(t)\right)=t\right\} .
$$


Then, take an orthonormal basis

$$
\left\{e_{1}, e_{2}, \cdots, e_{n-1}\right\}:=\left\{e_{1}(v), e_{2}(v), \cdots, e_{n-1}(v)\right\}
$$

of the hyperplane in $T_{p} M$ orthogonal to $e_{n}:=v$. Then, we denote by $E_{i}(t ; v)$, $i=1,2, \cdots, n$, parallel vector fields along $\gamma_{v}$ such that $E_{i}(0 ; v)=e_{i}$. Moreover, let $\sigma_{t}$ be a 2-dimensional linear subspace of $T_{\gamma_{v}(t)} M$ spanned by $\gamma_{v}^{\prime}(t)$ and a tangent vector $w_{t}$ to $M$ at $\gamma_{v}(t)$ defined by

$$
w_{t}:=\sum_{i=1}^{n-1} a_{i} E_{i}(t ; v)
$$

with

$$
\sum_{i=1}^{n-1} a_{i}^{2}=1
$$

for $a_{1}, a_{2}, \cdots, a_{n-1} \in \mathbb{R}$. Thus, the radial sectional curvature $K_{M}\left(\sigma_{t}\right)$ at $p$ of $M$ is given by

$$
K_{M}\left(\sigma_{t}\right)=\left\langle R\left(E_{n}(t ; v), w_{t}\right) E_{n}(t ; v), w_{t}\right\rangle,
$$

where $R$ denotes the Riemannian curvature tensor of $M$, which is a multi-linear map, defined by

$$
R(X, Y) Z:=\nabla_{Y} \nabla_{X} Z-\nabla_{X} \nabla_{Y} Z+\nabla_{[X, Y]} Z
$$

for smooth vector fields $X, Y, Z$ over $M$. Now, we set

$$
\boldsymbol{a}:=\left(\begin{array}{c}
a_{1} \\
a_{2} \\
\vdots \\
a_{n-1}
\end{array}\right) \in \mathbb{S}^{n-2}(1),
$$

where $\mathbb{S}^{n-2}(1) \subset \mathbb{R}^{n-1}$ is a standard unit sphere, and set

$$
R_{i j}(t, v):=\left\langle R\left(E_{n}(t ; v), E_{i}(t ; v)\right) E_{n}(t ; v), E_{i}(t ; v)\right\rangle .
$$

Observe that $R_{i j}(t, v)$ is a $C^{\infty}$-function. Then, by (5.1), we see that

$$
K_{M}\left(\sigma_{t}\right)=\sum_{i, j=1}^{n-1} a_{i} a_{j} R_{i j}(t, v)={ }^{t} \boldsymbol{a} R(t ; v) \boldsymbol{a},
$$

where $R(t ; v):=\left(R_{i j}(t, v)\right)$ is a symmetric $(n-1) \times(n-1)$-matrix. Furthermore, we set

$$
F_{0}(t, v):=\min \left\{{ }^{t} \boldsymbol{a} R(t ; v) \boldsymbol{a} \mid \boldsymbol{a} \in \mathbb{S}^{n-2}(1)\right\}
$$

for all $(t, v) \in[0, \infty) \times \mathbb{S}_{p}^{n-1}$ with $t \leq \rho(v)$. Thus, by (5.2), we get

$$
K_{M}\left(\sigma_{t}\right) \geq F_{0}(t, v)
$$

for all $(t, v) \in[0, \infty) \times \mathbb{S}_{p}^{n-1}$ with $t \leq \rho(v)$. Note that $F_{0}(t, v)$ is locally Lipschitz on $[0, \infty) \times \mathbb{S}_{p}^{n-1}$ with $t \leq \rho(v)$, since $R_{i j}(t, v)$ is a $C^{\infty}$-function.

We define

$$
F(t, v):=F_{0}\left(\rho_{t}(v), v\right)
$$


on $[0, \infty) \times \mathbb{S}_{p}^{n-1}$, where we set $\rho_{t}(v):=\min \{\rho(v), t\}$. Since $F(t, v)$ is locally Lipschitz on $[0, \infty) \times \mathbb{S}_{p}^{n-1}$ with $t \leq \rho(v)$, for any $b>0$, there exists a constant $C_{0}$ such that

$$
\left|F\left(t_{1}, v\right)-F\left(t_{2}, v\right)\right| \leq C_{0}\left|\rho_{t_{1}}(v)-\rho_{t_{2}}(v)\right|
$$

for all $t_{1}, t_{2} \in[0, b]$ and all $v \in \mathbb{S}_{p}^{n-1}$. Since it is clear that

$$
\left|\rho_{t_{1}}(v)-\rho_{t_{2}}(v)\right| \leq\left|t_{1}-t_{2}\right|
$$

for all $t_{1}, t_{2} \in[0, b]$ and all $v \in \mathbb{S}_{p}^{n-1}$, it follows from (5.4) that

$$
\left|F\left(t_{1}, v\right)-F\left(t_{2}, v\right)\right| \leq C_{0}\left|t_{1}-t_{2}\right|
$$

holds for all $t_{1}, t_{2} \in[0, b]$ and all $v \in \mathbb{S}_{p}^{n-1}$. Then, we get a locally Lipschitz function

$$
G(t):=\min \left\{F(t, v) \mid v \in \mathbb{S}_{p}^{n-1}\right\}
$$

on $[0, \infty)$. Indeed, take any $t_{1}, t_{2} \in[0, b]$. Without loss of generality, we may assume that $G\left(t_{1}\right) \geq G\left(t_{2}\right)$. By (5.5),

$$
\begin{aligned}
G\left(t_{1}\right)-G\left(t_{2}\right) & =G\left(t_{1}\right)-F\left(t_{2}, v_{2}\right) \\
& \leq F\left(t_{1}, v_{2}\right)-F\left(t_{2}, v_{2}\right) \leq C_{0}\left|t_{1}-t_{2}\right| .
\end{aligned}
$$

Here, $v_{2} \in \mathbb{S}_{p}^{n-1}$ is a point satisfying $G\left(t_{2}\right)=F\left(t_{2}, v_{2}\right)$. Thus, we have proved that $G(t)$ is locally Lipschitz on $[0, \infty)$. Therefore, it follows from (5.3) and (5.6) that

$$
K_{M}\left(\sigma_{t}\right) \geq G(t)
$$

holds.

5.2. Proof of partial answer to Milnor's open conjecture. Before starting the proof of our answer to Milnor's open conjecture, we will recall the following differential inequality (compare [Z, Lemma 2.1]).

Lemma 5.2. Let $\phi(t)$ be a $C^{1}$-function on $[0, \infty)$, and $\lambda(t)$ a continuous function on $[0, \infty)$. If

$$
\phi^{\prime}(t) \leq \lambda(t) \phi(t)
$$

holds on $[0, \infty)$, then we have

$$
\phi(t) \leq e^{\Lambda(t)} \phi(0) .
$$

Here, we set

$$
\Lambda(t):=\int_{0}^{t} \lambda(s) d s
$$

Proof. Since $0 \geq \phi^{\prime}(t)-\lambda(t) \phi(t)$ on $[0, \infty)$ by the assumption on this lemma, we see that

$$
0 \geq e^{-\Lambda(t)} \phi^{\prime}(t)-e^{-\Lambda(t)} \lambda(t) \phi(t)=\frac{d}{d t}\left(e^{-\Lambda(t)} \phi(t)\right)
$$

for all $t \in[0, \infty)$. Thus, by (5.7), we have

$$
\begin{aligned}
0=\int_{0}^{t} 0 d s & \geq \int_{0}^{t} \frac{d}{d s}\left(e^{-\Lambda(s)} \phi(s)\right) d s \\
& =e^{-\Lambda(t)} \phi(t)-e^{-\Lambda(0)} \phi(0) \\
& =e^{-\Lambda(t)} \phi(t)-\phi(0)
\end{aligned}
$$


for all $t \in[0, \infty)$. Therefore, by (5.8), we get

$$
e^{-\Lambda(t)} \phi(t) \leq \phi(0)
$$

that is, $\phi(t) \leq e^{\Lambda(t)} \phi(0)$ holds on $[0, \infty)$.

Now, let $G(t)$ be the Lipschitz function in the Model Lemma (Lemma 5.1), and set

$$
G^{*}(t):=\min \{0, G(t)\} .
$$

Consider a non-compact model surface of revolution $\left(M^{*}, p^{*}\right)$ with its metric

$$
g^{*}=d t^{2}+m(t)^{2} d \theta^{2}, \quad(t, \theta) \in(0, \infty) \times \mathbb{S}_{p^{*}}^{1}
$$

satisfying the differential equation $m^{\prime \prime}(t)+G^{*}(t) m(t)=0$ with initial conditions $m(0)=0$ and $m^{\prime}(0)=1$. Note that the metric (5.9) is not always differentiable around the base point $p^{*} \in M^{*}$.

Theorem 5.3. Let $M$ be a complete open Riemannian $n$-manifold, $p \in M$ any fixed point, and $\left(M^{*}, p^{*}\right)$ a comparison model surface of revolution, constructed from $(M, p)$, with its metric (5.9). If $G^{*}(t)$ satisfies

$$
\int_{0}^{\infty}\left(-t \cdot G^{*}(t)\right) d t<\infty
$$

then the total curvature $c\left(M^{*}\right)$ is finite. In particular, then $M$ has a finitely generated fundamental group.

Proof. Since $G^{*}(t) \leq 0$ on $[0, \infty)$, we have

$$
m^{\prime \prime}(t)=-G^{*}(t) m(t) \geq 0
$$

on $[0, \infty)$; that is, $m^{\prime}(t)$ is increasing on $[0, \infty)$. Since $m^{\prime}(0)=1$,

$$
m^{\prime}(t) \geq m^{\prime}(0)=1
$$

holds for all $t \in[0, \infty)$. In particular, $m(t)$ is non-negative for all $t \geq 0$, for $m(t)$ is increasing on $[0, \infty)$ and $m(0)=0$. We will first prove that the total curvature $c\left(M^{*}\right)$ of $\left(M^{*}, p^{*}\right)$ is finite. To show this fact, we define

$$
\phi(t):=m^{\prime}(t)
$$

on $[0, \infty)$. Then, since $m(0)=0$ and $m^{\prime}(t)$ is increasing on $[0, \infty)$, we see that

$$
\frac{m(t)}{t}=\frac{1}{t} \int_{0}^{t} m^{\prime}(s) d s \leq \frac{m^{\prime}(t)}{t} \int_{0}^{t} d s=\phi(t)
$$

on $(0, \infty)$. Thus, by (5.11), we have

$$
\begin{aligned}
\phi^{\prime}(t)=m^{\prime \prime}(t) & =-G^{*}(t) m(t) \\
& =\left(-t G^{*}(t)\right) \cdot \frac{m(t)}{t} \\
& \leq-t G^{*}(t) \phi(t) \\
& =\lambda(t) \phi(t)
\end{aligned}
$$

for all $t \in(0, \infty)$. Here, we set $\lambda(t):=-t G^{*}(t)(\geq 0)$. In particular,

$$
\phi^{\prime}(t) \leq \lambda(t) \phi(t)
$$


holds on $[0, \infty)$, since $G^{*}(t)$ is continuous on $[0, \infty)$. Then, it follows from Lemma 5.2 and $m^{\prime}(0)=1$ that we have

$$
\phi(t) \leq e^{\Lambda(t)} \phi(0)=e^{\Lambda(t)} m^{\prime}(0)=e^{\Lambda(t)}
$$

on $[0, \infty)$. Here, we set

Since

$$
\Lambda(t):=\int_{0}^{t} \lambda(s) d s
$$

$$
0 \leq \Lambda(\infty)=\int_{0}^{\infty} \lambda(s) d s=\int_{0}^{\infty}\left(-s \cdot G^{*}(s)\right) d s<\infty
$$

by the assumption (5.10), we see, by (5.12), that

$$
\lim _{t \rightarrow \infty} m^{\prime}(t)=\lim _{t \rightarrow \infty} \phi(t) \leq \lim _{t \rightarrow \infty} e^{\Lambda(t)}=e^{\Lambda(\infty)}<\infty .
$$

By (5.13), we get

$$
c\left(M^{*}\right)=2 \pi \int_{0}^{\infty}\left(-m^{\prime \prime}(t)\right) d t=2 \pi\left(1-\lim _{t \rightarrow \infty} m^{\prime}(t)\right)>-\infty .
$$

Thus, $c\left(M^{*}\right)$ is finite.

Therefore, it follows from the Sector Theorem and the Main Theorem that $M$ has finite topological type; that is, $M$ is homeomorphic to the interior of a compact manifold with boundary. In particular, $M$ therefore has a finitely generated fundamental group.

Finally, we prove a corollary to our Main Theorem, which is another partial answer to Milnor's open conjecture.

Let $K_{M}$ be the sectional curvature of an arbitrary complete Riemannian $n$ manifold $M$. Furthermore, we define a (real) number $K_{M}(q)$ as follows:

$$
K_{M}(q):=\min _{\sigma \subset T_{q} M} K_{M}(\sigma) .
$$

Here, $\sigma \subset T_{q} M$ is a 2-dimensional linear space, and $K_{M}(\sigma)$ is the sectional curvature of $\sigma$ at $q \in M$.

Corollary 5.4. Let $M$ be an arbitrary complete open Riemannian n-manifold, $p \in M$ any fixed point, and $\left(M^{*}, p^{*}\right)$ a comparison model surface of revolution, constructed from $(M, p)$, with its metric (5.9). If the sectional curvature $K_{M}$ of $M$ satisfies

$$
\liminf _{t \rightarrow \infty} t^{2+\alpha} \min _{q \in B_{t}(p)} K_{M}(q)>\mathcal{N}
$$

for some numbers $\alpha>0$ and $\mathcal{N} \leq 0$, then $M$ has a finitely generated fundamental group.

Proof. By the assumption (5.14), we have

$$
t^{2+\alpha} \min _{q \in B_{t}(p)} K_{M}(q)>C \cdot \mathcal{N}
$$

for some $C \geq 1$ and all $t>1$. Then, it follows from the construction of $G(t)$ (see the proof of Lemma 5.1) and (5.15) that

$$
G(t) \geq \min _{q \in B_{t}(p)} K_{M}(q)>\frac{C \cdot \mathcal{N}}{t^{2+\alpha}}
$$


holds for all $t>1$. Since $G^{*}(t) \leq 0$ on $[0, \infty)$, we see, by (5.16), that

$$
0 \geq \int_{1}^{\infty} t \cdot G^{*}(t) d t \geq \int_{1}^{\infty} \frac{C \cdot \mathcal{N}}{t^{1+\alpha}} d t=\left[-\frac{C \cdot \mathcal{N}}{\alpha t^{\alpha}}\right]_{1}^{\infty}=\frac{C \cdot \mathcal{N}}{\alpha}>-\infty
$$

Hence, we get, by (5.17),

$$
0 \leq \int_{0}^{\infty}-t \cdot G^{*}(t) d t<\infty
$$

Therefore, by the Sector Theorem and the Main Theorem, $M$ has a finitely generated fundamental group.

Remark 5.5. Under the assumption in Theorem 5.3, or Corollary 5.4, it follows from [MNO, Theorem 0.1] that $(M, p)$ admits the asymptotic cone via rescaling argument; i.e., the pointed Gromov-Hausdorff limit space of $((1 / t) M, p)$ exists as $t \rightarrow \infty$, and the space is, naturally, isometric to a Euclidean cone (see G2, Definition 3.14] for a definition of the pointed Gromov-Hausdorff convergence). However, one should notice again that our models in Theorem 5.3 and Corollary 5.4 have been constructed from any complete open Riemannian manifold with an arbitrary given point as a base point, and that the metrics (5.9) in Theorem 5.3 and Corollary 5.4 are not always differentiable around their base points. In particular, our Main Theorem has a wider class of metrics than those described in MNO, Theorem 0.1].

The next example shows that non-negative radial curvature does not always mean non-negative sectional curvature.

Example 5.6. Let $M$ be a 2 -sphere of revolution with a Riemannian metric

$$
h:=d r^{2}+m(r)^{2} d \theta^{2}, \quad(r, \theta) \in(0,2 a) \times \mathbb{S}_{p}^{1}
$$

and pair of poles $p, q$; i.e., the surface $(M, h)$ satisfies that

(TS-1) $(M, h)$ is symmetric with respect to the reflection fixing $r=a$, where $2 a=d(p, q)$,

(TS-2) the radial curvature function $G \circ \gamma:[0,2 a] \longrightarrow \mathbb{R}$ of $M$ is monotonic along a meridian $\gamma$ emanating from $p=\gamma(0)$ to the point on $r=a$.

Note that $(M, h)$ does not always have a positive radial curvature function $G \circ \gamma(t)$. For example, the model surface of revolution generated by the $(x, z)$-plane curve $(m(r), 0, z(r))$ satisfies (TS-1) and (TS-2), where

$$
m(r):=\frac{\sqrt{3}}{10}\left(9 \sin \frac{\sqrt{3}}{9} r+7 \sin \frac{\sqrt{3}}{3} r\right), \quad z(r):=\int_{0}^{r} \sqrt{1-m^{\prime}(t)^{2}} d t .
$$

In particular, we then see that $G(\gamma(3 \sqrt{3} \pi / 2))=-1$ (see [SiT2]).

Thus, without loss of generality, by setting $2 a:=\pi$, we may assume that (5.18) is the geodesic polar coordinates around the north pole $(0,0,1)$ of the unit sphere $\mathbb{S}^{2}(1)$ in 3-dimensional Euclidean space $\mathbb{R}^{3}$, and that the radial curvature function $G \circ \gamma(t)$ of $(M, h)$ is negative at a point on $(0, \pi)$. Now, we will introduce a new Riemannian metric $g$ on 3-dimensional Euclidean space $\left(\mathbb{R}^{3}, g_{0}\right)$. Outside of the unit ball $B_{1}(o) \subset \mathbb{R}^{3}$ centered at the origin $o \in \mathbb{R}^{3}$, we define $g$ to be

$$
g:=d t^{2}+f(t)^{2} h,
$$

where $f:(0, \infty) \longrightarrow(0, \infty)$ is a smooth function, and the function $t$ denotes the Euclidean distance function from $o \in \mathbb{R}^{3}$. We set $x_{1}:=t, x_{2}:=r$, and $x_{3}:=\theta$, 
and denote by $\sigma_{i j}$ a 2-dimensional linear plane spanned by $\partial / \partial x_{i}$ and $\partial / \partial x_{j}, i \neq j$. Then, the sectional curvatures $K\left(\sigma_{i j}\right)$ of the planes $\sigma_{i j}$ at each point on $\mathbb{R}^{3} \backslash B_{1}(o)$ are

$$
K\left(\sigma_{12}\right)=K\left(\sigma_{13}\right)=-\frac{f^{\prime \prime}(t)}{f(t)}
$$

and

$$
K\left(\sigma_{23}\right)=\frac{1}{f(t)^{2}}\left(-\frac{m^{\prime \prime}(r)}{m(r)}-f^{\prime}(t)^{2}\right) .
$$

Consider a smooth family $\left\{h_{t}\right\}_{t \geq 0}$ of Riemannian metrics such that $h_{t}$ is the standard metric on $\mathbb{S}^{2}(1)$ for small $t$ and $h_{t}=h$ for $t \geq 1$. Then, $(t, r, \theta)$ are the geodesic polar coordinates around $o \in \mathbb{R}^{3}$ for $\left(\mathbb{R}^{3}, g_{0}\right)$, and the Riemannian metric $g_{t}:=d t^{2}+f(t)^{2} h_{t}$ on $\mathbb{R}^{3}$ equals $g_{0}$ for small $t$ if $f(t)=t$, and equals $g$ on $\mathbb{R}^{3} \backslash B_{1}(o)$. By the definition of $g_{t}$, each $t$-curve on $\left(\mathbb{R}^{3}, g_{t}\right)$ is a ray emanating from $o \in \mathbb{R}^{3}$. Therefore, it follows from (5.19) and (5.20) that the radial curvature of $\left(\mathbb{R}^{3}, g\right)$ is non-negative on $\mathbb{R}^{3} \backslash B_{1}(o)$ if $f^{\prime \prime}(t) \leq 0$. In particular, $\left(\mathbb{R}^{3}, g\right)$ admits non-negative Ricci curvature at divergent points.

From Example 5.6. we may conclude that Milnor's open conjecture does not follow from our Main Theorem, and, at the same time, our Main Theorem does not follow from Milnor's open conjecture. Moreover, we find that the radial curvature geometry deals with a geometry different from the geometry of a global lower bound on Ricci curvature, but with a sufficiently large geometry.

\section{ACKNOWLEDGEMEnTS}

The first-named author would like to express to Professor Karsten Grove the deepest gratitude for his encouragement and his interest in our work. We are very grateful to Professors Takashi Shioya and Shin-ichi Ohta for their helpful comments on the first draft of this article. Finally, we also thank the referee for a careful reading of the manuscript, valuable suggestions, and helpful comments on the manuscript, which, no doubt, have improved the presentation of this article.

\section{REFERENCES}

[A] U. Abresch, Lower curvature bounds, Toponogov's theorem, and bounded topology, Ann. Sci. École Norm. Sup. (4) 18 (1985), 651-670. MR839689 (87j:53058)

[AG] U. Abresch and D. Gromoll, On complete manifolds with nonnegative Ricci curvature, J. Amer. Math. Soc. 3 (1990), No. 2, 355-374. MR1030656 (91a:53071)

[Al] A. D. Aleksandrov, Die Innere Geometrie der Konvexen Flächen, Akademie Verlag, Berlin, 1955. MR0071041 (17:74d)

[B] M. Berger, Les variétés riemannienes (1/4)-pincées, Ann. Scuola Norm. Sup. Pisa (3) 14 (1960), 161-170. MR0140054 (25:3478)

[Bo] O. Bonnet, Sur quelques propriétés des lignes géodésiques, Comptes Rendus Ac. Sc. Paris 40 (1855), 1311-1313.

[BGP] Yu. D. Burago, M. Gromov, and G. Ya. Perel'man, A. D. Aleksandrov spaces with curvatures bounded below, Uspekhi Mat. Nauk 47 (1992), No. 2, 3-51, translation in Russian Math. Surveys 47 (1992), No. 2, 1-58. MR1185284 (93m:53035)

[CE] J. Cheeger and D. G. Ebin, Comparison Theorems in Riemannian Geometry, NorthHolland Mathematical Library, vol. 9, North-Holland, Amsterdam, 1975. MR0458335 $(56: 16538)$

[CG] J. Cheeger and D. Gromoll, On the structure of complete manifolds of nonnegative curvature, Ann. of Math. (2) 96 (1972), 415-443. MR0309010(46:8121) 
[CV] S. Cohn-Vossen, Totalkrümmung und geodätische Linien auf einfach zusammenhängenden offenen volständigen Flächenstücken, Recueil Math. Moscow 43 (1936), 139163.

[D] U. Dini, Fondamenti per la teorica delle funzioni di variabili reali, Pisa, 1878.

[GlSi] H. Gluck and D. A. Singer, Scattering of geodesic fields, II, Ann. of Math. (2) 110 (1979), 205-225. MR.549487 (80k:53073)

[GMST] J. Gravesen, S. Markvorsen, R. Sinclair, and M. Tanaka, The cut locus of a torus of revolution, Asian J. Math. 9 (2005), 103-120. MR2150694 (2006b:53042)

[G1] M. Gromov, Curvature, diameter and Betti numbers, Comment. Math. Helv. 56 (1981), 179-195. MR630949 (82k:53062)

[G2] M. Gromov, Metric Structures for Riemannian and non-Riemannian Spaces, Birkhäuser, Boston, MA, 1999. Based on the 1981 French original (MR 85e:53051), with appendices by M. Gromov, M. Katz, P. Pansu and S. Semmes; translated from the French by Sean Michael Bates. MR 2307192 (2007k:53049)

[GP] K. Grove and P. Peterson, Bounding homotopy type by geometry, Ann. of Math. (2) 128 (1988), 195-206. MR951512 (90a:53044)

[GPW] K. Grove, P. Peterson, and J. Wu, Geometric finiteness theorems via controlled topology, Invent. Math. (2) 99 (1990), 205-213; erratum, 104 (1991), 221-222. MR1029396 (90k:53075) MR:1094053(92b:53065)

[GS] K. Grove and K. Shiohama, A generalized sphere theorem, Ann. of Math. (2) 106 (1977), 201-211. MR0500705 (58:18268)

[Hr] P. Hartman, Ordinary Differential Equations (the second edition), Classics in Applied Mathematics 38, Society for Industrial and Applied Mathematics, Philadelphia, 1982. MR1929104 (2003h:34001)

[Hw] T. Hawkins, Lebesgue's Theory of Integration : Its origins and development, University of Wisconsin Press, Madison, 1970. MR0269473 (42:4368)

[He] J. J. Hebda, Metric structure of cut loci in surfaces and Ambrose's problem, J. Differential Geom. 40 (1994), 621-642. MR1305983 (95m:53046)

[IT] J. Itoh and M. Tanaka, The Lipschitz continuity of the distance function to the cut locus, Trans. Amer. Math. Soc. 353 (2001), 21-40. MR1695025 (2001b:53029)

[IMS] Y. Itokawa, Y. Machigashira, and K. Shiohama, Generalized Toponogov's theorem for manifolds with radial curvature bounded below, Explorations in complex and Riemannian geometry, 121-130, Contemp. Math. 332, Amer. Math. Soc., Providence, RI, 2003. MR.2018335 (2004j:53051)

[KK] N. N. Katz and K. Kondo, Generalized space forms, Trans. Amer. Math. Soc. 354 (2002), 2279-2284. MR1885652 (2002k:53064)

[K1] W. Klingenberg, Contributions to Riemannian geometry in the large, Ann. of Math. (2) 69 (1959), 654-666. MR0105709 (21:4445)

[K2] W. Klingenberg, Über Riemannsche Mannigfaltigkeiten mit positiver Krümmung, Comm. Math. Helv. 35 (1961), 47-54. MR0139120(25:2559)

[KO] K. Kondo and S. Ohta, Topology of complete manifolds with radial curvature bounded from below, Geom. Funct. Anal. 17 (2007), 1237-1247. MR2373016 (2008k:53069)

[KT1] K. Kondo and M. Tanaka, Total curvatures of model surfaces control topology of complete open manifolds with radial curvature bounded below. I, Preprint 2006, submitted to Math. Ann. (October 23, 2006), http://arXiv.org/abs/0901.4010

[KT2] K. Kondo and M. Tanaka, Toponogov comparison theorem for open triangles, Preprint 2009, http://arXiv.org/abs/0901.4019

[Ma] H. von Mangoldt, Über diejenigen Punkte auf positiv gekrümmten Flächen, welche die Eigenschaft haben, dass die von Ihnen ausgehenden geodätischen Linien nie aufhören, kürzeste Linien zu sein, J. Reine. Angew. Math. 91 (1881), 23-53.

[MNO] Y. Mashiko, K. Nagano, and K. Otsuka, The asymptotic cones of manifolds of roughly non-negative radial curvature, J. Math. Soc. Japan 57 (2005), 55-68. MR2114720 (2006e:53070)

[Mi] J. Milnor, A note on curvature and the fundamental group, J. Differential Geom. 2 (1968), 1-7. MR0232311 (38:636)

[O1] S. Ohta, Markov type of Alexandrov spaces of non-negative curvature, Mathematika 55 (2009), 177-189. MR2573607 
[O2] S. Ohta, Gradient flows on Wasserstein spaces over compact Alexandrov spaces, Amer. J. Math. 131 (2009), 475-516. MR.2503990

[O3] S. Ohta, Uniform convexity and smoothness, and their applications in Finsler geometry, Math. Ann. 343 (2009), 669-699. MR2480707 (2009m:53199)

[OS] Y. Otsu and T. Shioya, The Riemannian structure of Alexandrov spaces, J. Differential Geom. 39 (1994), 629-658. MR1274133 (95e:53062)

[P1] G. Ya. Perel'man, A. D. Aleksandrov spaces with curvatures bounded below, II, Preprint, 1991.

[P2] G. Ya. Perel'man, Elements of Morse theory on Aleksandrov spaces, St. Petersburg Math. J. 5 (1994), 205-213. MR1220498 (94h:53054)

[Po] H. Poincaré, Sur les lignes géodésiques des surfaces convexes, Trans. Amer. Math. Soc. 6 (1905), 237-274. MR.1500710

[R] H. E. Rauch, A contribution to differential geometry in the large, Ann. of Math. (2) 54 (1951), 38-55. MR0042765 (13:159b)

[Sa] T. Sakai, Riemannian Geometry, Translations of Mathematical Monographs 149, American Mathematical Society, Providence, RI, 1996. MR1390760 (97f:53001)

[Sc] I. J. Schoenberg, Some applications of the calculus of variations to Riemannian geometry, Ann. of Math. (2) 33 (1932), 485-495. MR1503071

[Sh1] K. Shiohama, The role of total curvature on complete noncompact Riemannian 2manifolds, Illinois J. Math. 28 (1984), 597-620. MR761993 (86h:53069)

[Sh2] K. Shiohama, Recent developments in sphere theorems, Proc. Symp. Pure Math. 54, 551576, Amer. Math. Soc., 1993. MR1216646 (94d:53071)

[SST] K. Shiohama, T. Shioya, and M. Tanaka, The Geometry of Total Curvature on Complete Open Surfaces, Cambridge Tracts in Mathematics 159, Cambridge University Press, Cambridge, 2003. MR2028047 (2005c:53037)

[ST1] K. Shiohama and M. Tanaka, Cut loci and distance spheres on Alexandrov surfaces, Séminaires \& Congrès, Collection Soc. Math. France, No.1, Actes de la table ronde de Géométrie différentielle en l'honneur Marcel Berger, (1996), 531-560. MR:1427770 (98a:53062)

[ST2] K. Shiohama and M. Tanaka, Compactification and maximal diameter theorem for noncompact manifolds with radial curvature bounded below, Math. Zeitschrift 241 (2002), 341-351. MR1935490 (2003i:53048)

[SY1] T. Shioya and T. Yamaguchi, Collapsing three-manifolds under a lower curvature bound, J. Differential Geom. 56 (2000), No. 1, 1-66. MR.1863020 (2002k:53074)

[SY2] T. Shioya and T. Yamaguchi, Volume collapsed three-manifolds with a lower curvature bound, Math. Ann. 333 (2005), 131-155. MR2169831 (2006j:53050)

[SiT1] R. Sinclair and M. Tanaka, A bound on the number of endpoints of the cut locus, London Math. Soc., J. Comput. Math. 9 (2006), 21-39. MR2199583 (2006i:53039)

[SiT2] R. Sinclair and M. Tanaka, The cut locus of a two-sphere of revolution and Toponogov's comparison theorem, Tohoku Math. J. 59 (2007), 379-399. MR2365347(2008k:53075)

[So] C. Sormani, Nonnegative Ricci curvature, small linear diameter growth, and finite generation of fundamental groups, J. Differential Geom. 54 (2000), No. 3, 547-559. MR.1823314 (2003a:53047)

[St] C. Sturm, Sur les équations différentielles linéaires du second ordre, J. Math. Pures Appl. (1) 1 (1836), 106-186.

[T1] M. Tanaka, On the cut loci of a von Mangoldt's surface of revolution, J. Math. Soc. Japan 44 (1992), 631-641. MR:1180440 (93h:53034)

[T2] M. Tanaka, On a characterization of a surface of revolution with many poles, Mem. Fac. Sci., Kyushu Univ. Ser. A, Mathematics 46 (1992), 251-268. MR1195469 (93m:53033)

[To1] V.A. Toponogov, Riemann spaces having their curvature bounded below by a positive number (in Russian), Dokl. Akad. Nauk SSSR 120 (1958), 719-721. MR0099701 $(20: 6139)$

[To2] V.A. Toponogov, Riemann spaces with curvature bounded below (in Russian), Uspehi Mat. Nauk 14 (1959), no. 1 (85), 87-130. MR0103510 (21:2278)

[WZ] R. L. Wheeden and A. Zygmund, Measure and Integral, Marcel Dekker, New York, 1977. MR.0492146 (58:11295)

[W] B. Wilking, On fundamental groups of manifolds of nonnegative curvature, Differential Geom. Appl. 13 (2000), no. 2, 129-165. MR1783960 (2001g:53076) 
[Y] T. Yamaguchi, A convergence theorem in the geometry of Alexandrov spaces, Séminaires \& Congrès, Collection Soc. Math. France, No.1, Actes de la table ronde de Géométrie différentielle en l'honneur Marcel Berger, (1996), 601-642. MR1427772 (97m:53078)

[Z] S.-H. Zhu, A volume comparison theorem for manifolds with asymptotically nonnegative curvature and its applications, Amer. J. Math. 116 (1994), 669-682. MR.1277451 (95c:53049)

Department of Mathematics, Tokai University, Hiratsuka City, Kanagawa Pref. $259-1292$ JAPAN

E-mail address: keikondo@keyaki.cc.u-tokai.ac.jp

Department of Mathematics, Tokai University, Hiratsuka City, Kanagawa Pref. $259-1292$ JAPAN

E-mail address: m-tanaka@sm.u-tokai.ac.jp 